\title{
BicPAM: Pattern-based biclustering for biomedical data analysis
}

\author{
Rui Henriques* ${ }^{*}$ and Sara C Madeira
}

\begin{abstract}
Background: Biclustering, the discovery of sets of objects with a coherent pattern across a subset of conditions, is a critical task to study a wide-set of biomedical problems, where molecular units or patients are meaningfully related with a set of properties. The challenging combinatorial nature of this task led to the development of approaches with restrictions on the allowed type, number and quality of biclusters. Contrasting, recent biclustering approaches relying on pattern mining methods can exhaustively discover flexible structures of robust biclusters. However, these approaches are only prepared to discover constant biclusters and their underlying contributions remain dispersed.

Methods: The proposed BicPAM biclustering approach integrates existing principles made available by state-of-the-art pattern-based approaches with two new contributions. First, BicPAM is the first efficient attempt to exhaustively mine non-constant types of biclusters, including additive and multiplicative coherencies in the presence or absence of symmetries. Second, BicPAM provides strategies to effectively compose different biclustering structures and to handle arbitrary levels of noise inherent to data and with discretization procedures.
\end{abstract}

Results: Results show BicPAM's superiority against its peers and its ability to retrieve unique types of biclusters of interest, to efficiently deliver exhaustive solutions and to successfully recover planted biclusters in datasets with varying levels of missing values and noise. Its application over gene expression data leads to unique solutions with heightened biological relevance.

Conclusions: BicPAM approaches integrate existing disperse efforts towards pattern-based biclustering and provides the first critical strategies to efficiently discover exhaustive solutions of biclusters with shifting, scaling and symmetric assumptions with varying quality and underlying structures. Additionally, BicPAM dynamically adapts its behavior to mine data with different levels of missing values and noise.

Keywords: Biclustering, Pattern mining, Biomedical data analysis

\section{Introduction}

Biclustering, a local approach for clustering, seeks to find sub-matrices (biclusters), subsets of rows with a highly correlated expression pattern across a subset of columns. Biclustering has been extensively applied in gene expression data analysis [1], since small groups of genes can participate in multiple cellular processes or pathways of interest that may be only active in a subset of the conditions under analysis. Biclustering has been also applied to group mutations and copy number variations [2], to analyze biological networks [3], and to study translational [4], chemical [5] or nutritional data [6].

*Correspondence: rmch@tecnico.ulisboa.pt

INESC-ID and Instituto Superior Técnico, Universidade de Lisboa, Lisbon, Portugal
Biclustering involves hard combinatorial optimization. In particular, its complexity increases when rows and columns are allowed to participate in more than one bicluster (non-exclusive structure) and in no bicluster at all (non-exhaustive structure). Hence most existing algorithms are either based on greedy or stochastic approaches $[1,2,7,8]$, potentially producing sub-optimal solutions, or on finding a constrained number, structure or type of biclusters $[1,2,9]$.

The state-of-the-art attempts to tackle biclustering using pattern mining techniques allow for exhaustive and flexible searches and show solid levels of efficiency $[10,11]$. The fact that pattern mining research is driven by scalability requirements [12], opens a critical direction to

(c) 2014 Henriques and Madeira; licensee BioMed Central. This is an Open Access article distributed under the terms of the Creative Commons Attribution License (http://creativecommons.org/licenses/by/4.0), which permits unrestricted use, distribution, and reproduction in any medium, provided the original work is properly credited. The Creative Commons Public Domain Dedication waiver (http://creativecommons.org/publicdomain/zero/1.0/) applies to the data made available in this article, unless otherwise stated. 
perform biclustering. Interestingly, the existing patternbased approaches for biclustering - such as BiModule [13], DeBi [10], RAP [14] and GenMiner [15] - provide complementary principles of interest for this field. However, these principles are not yet integrated. Additionally, existing approaches only discover biclusters with constant profiles $[10,13,14]$, and are not able to handle missing values or medium to high levels of noise. This work aims to target these limitations by proposing a pattern-based biclustering approach, BicPAM, that is able to combine existing potentialities from state-ofthe-art pattern-based approaches with two critical novel contributions:

- flexible exhaustive solutions: arbitrary number of (potentially overlapping) biclusters with additive, multiplicative and symmetric assumptions using multiple ranges of values;

- biclustering behavior dynamically adapted to deal with varying levels of noise and missing values.

To our knowledge, this is the first biclustering approach that is able to support and combine each of these two contributions. The importance of these contributions is shown experimentally over synthetic and biological data. Additionally, experimental results on both synthetic and real datasets demonstrate the efficiency and effectiveness of the pattern-based biclustering algorithms proposed in BicPAM.

The paper is organized as follows. Background covers essential concepts from biclustering and pattern mining, and surveys the contributions from existing pattern-based biclustering approaches. BicPAM: pattern-based biclustering describes the proposed algorithms. In Results, we assess BicPAM's performance on synthetic and real data. Finally, the contributions and implications of this work are synthesized.

\section{Background}

This section introduces fundamental concepts of biclustering and pattern mining, and surveys the related work on pattern-based biclustering.

Definition 1. Given a matrix, $A=(X, Y)$, with a set of rows $X=\left\{x_{1}, . ., x_{n}\right\}$, columns $Y=\left\{y_{1}, . ., y_{m}\right\}$, and elements $a_{i j} \in \mathbb{R}$ relating row $i$ and column $j$ :

- A bicluster $B=(I, J)$ is a $r \times s$ submatrix of $A$, where $I=\left(i_{1}, . ., i_{r}\right) \subset X$ is a subset of rows and $J=\left(j_{1}, . ., j_{s}\right) \subset Y$ is a subset of columns;

- The biclustering task is to identify a set of biclusters $\mathcal{B}=\left\{B_{1}, . ., B_{p}\right\}$ such that each bicluster $B_{k}=\left(I_{k}, J_{k}\right)$ satisfies specific criteria of homogeneity, where $I_{k} \subset X, J_{k} \subset Y$, and $k \in \mathbb{N}$.
Approaches to solve the biclustering task either explicitly or implicitly rely on a merit function to define the homogeneity criteria. An illustrative function is the variance of bicluster's values. Merit functions either guarantee intra-bicluster homogeneity, the overall homogeneity of the output set of biclusters (inter-bicluster homogeneity), or both. When combined within specific search procedures, merit functions are to define the type, quality and structure of biclustering solutions [1].

Merit functions can be defined to locally maximize greedy iterative searches $[7,8,16-19]$, to combine row- and column-based clusters [20-22], to exploit matrices recursively [23], and to stochastically model the target solution $[6,24]$. In exhaustive searches, which commonly rely on constrained formulations, merit functions are the heuristics that guide the space exploration $[9,25]$.

Figure 1 presents different types and structures of biclusters. Biclusters can follow constant or more flexible models, with coherency on rows or columns [1]. Biclusters under an additive-multiplicative model, also referred as shifting-scaling biclusters, can be discovered using merit functions based on $\delta$-offsets of noise [17,25], on vector-angle cosines [21], or on generative models of linear dependencies [2]. Biclusters with symmetries can be discovered by differential biclustering methods $[9,26]$ and by few others [14]. Additionally, plaid [6] and order-preserving [19] types of biclusters have also been tackled [27,28]. Multiple biclustering structures have been proposed [1], with some approaches constraining them to exhaustive, exclusive, non-overlapping structures, and others allowing more flexible structures with arbitrarily positioned overlapping biclusters [7].

\section{Pattern mining}

Patterns are itemsets, rules or substructures that appear in a dataset with frequency no less than a specified threshold. Finding patterns is critical to derive relations from data.

Definition 2. Let $\mathcal{L}$ be a finite set of items, and $P$ be an itemset $P \subseteq \mathcal{L}$. A transaction $t$ is a pair $\left(t_{i d}, P\right)$ with id $\in \mathbb{N}$. An itemset database $D$ over $\mathcal{L}$ is a finite set of transactions $\left\{t_{1}, . ., t_{n}\right\}$.

Definition 3. A transaction $\left(t_{i d}, P\right)$ contains $P^{\prime}$, denoted $P^{\prime} \subseteq\left(t_{i d}, P\right)$, if $P^{\prime} \subseteq P$. The coverage $\Phi_{P}$ of an itemset $P$ is the set of all transactions in $D$ in which the itemset $P$ occurs: $\Phi_{P}=\{t \in D \mid P \subseteq t\}$. The support of an itemset $P$ in $D$,

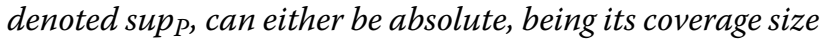
$\left|\Phi_{P}\right|$, or a relative threshold given by $\left|\Phi_{P}\right| /|D|$.

Definition 4. Given an itemset database $D$ and a minimum support threshold $\theta$, the frequent itemset mining (FIM) problem consists of computing the set $\{P \mid P \subseteq$ $\left.\mathcal{L}, \sup _{P} \geq \theta\right\}$. 


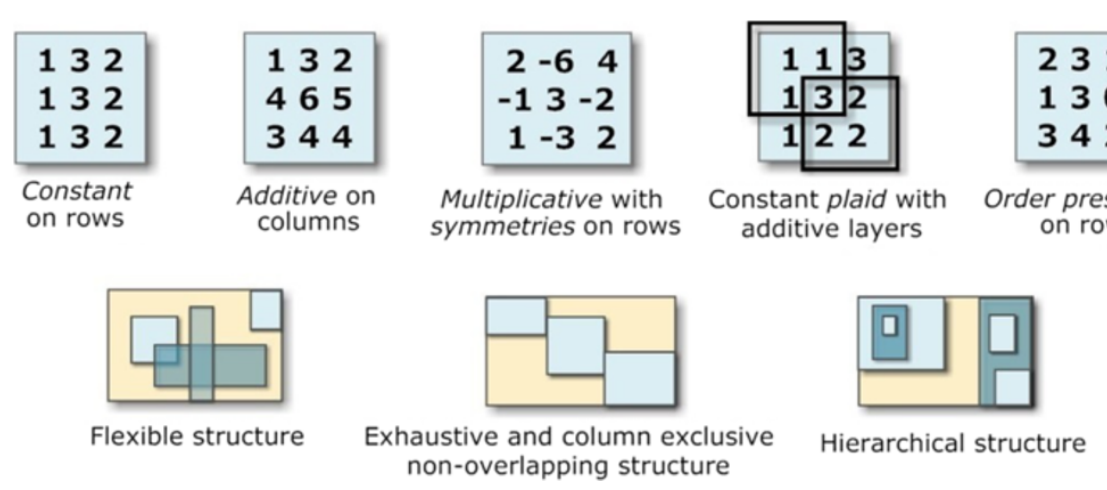

Figure 1 Illustrative bicluster types and biclustering structures.

A frequent itemset is an itemset with $\sup _{P} \geq \theta$. An accepted pattern is a frequent itemset that satisfies any other placed constraints over $D$.

To illustrate these concepts, consider the following itemset database, $\mathrm{D}_{e x}=\left\{\left(\mathrm{t}_{1},\{\mathrm{~B}, \mathrm{E}, \mathrm{G}\}\right),\left(\mathrm{t}_{2},\{\mathrm{~A}, \mathrm{~B}, \mathrm{C}, \mathrm{E}, \mathrm{H}, \mathrm{J}\}\right)\right.$, $\left.\left(\mathrm{t}_{3},\{\mathrm{~A}, \mathrm{~B}, \mathrm{D}, \mathrm{H}, \mathrm{J}\}\right),\left(\mathrm{t}_{4},\{\mathrm{D}, \mathrm{H}, \mathrm{J}\}\right),\left(\mathrm{t}_{5},\{\mathrm{~A}, \mathrm{H}, \mathrm{J}\}\right),\left(\mathrm{t}_{6},\{\mathrm{~A}, \mathrm{G}\}\right)\right\}$. We have $|\mathcal{L}|=|\{A, . ., J\}|=10, \Phi_{\{B, J\}}=\left\{t_{2}, t_{3}\right\}$ and $\sup _{\{B, J\}}=$ $\left|\left\{t_{2}, t_{3}\right\}\right| / 6=0 .(3)$. For $\theta=4$, FIM tasks returns $\{\{A\},\{H\},\{J\},\{H, J\}\}$.

Since FIM proposal [29], multiple extensions have been proposed, ranging from scalable data mining methodologies to multiple condensed and approximated pattern representations.

Definition 5. Given an itemset matrix, a support threshold $\theta$, and the coverage function $\Phi: 2^{\mathcal{L}} \rightarrow 2^{D}$ that maps an itemset $P$ to its set of supporting transactions:

- A frequent itemset $P$ is an itemset that satisfies $|\Phi(P)| \geq \theta$;

- A closed frequent itemset is a frequent itemset with no superset with same support $\left(\forall_{P^{\prime} \supset P}\left|P^{\prime}\right|<|P|\right)$;

- A maximal frequent itemset is a frequent itemset with all supersents being infrequent, $\forall_{P^{\prime} \supset P}\left|\Phi\left(P^{\prime}\right)\right|<\theta$.

A frequent itemset is maximal if all its supersets are infrequent, while it is closed if it is not a subset of an itemset with the same support. Considering the previously introduced itemset database $\mathrm{D}_{\text {ex }}$, a given threshold $\theta=3$ and $|P| \geq 2$, there is one maximal frequent itemset $(\{A, H, J\})$ and there are two closed frequent itemsets $(\{A, H, J\}$ and $\{H, J\})$.

Definition 6. Consider two itemsets $P \in 2^{\mathcal{L}}$ and $P^{\prime} \in$ $2^{\mathcal{L}}$, where $P^{\prime} \subseteq P$, and a predicate $M$. $M$ is monotonic when $M(P) \Rightarrow M\left(P^{\prime}\right)$ and $M$ is anti-monotonic when $\neg M\left(P^{\prime}\right) \Rightarrow$ $\neg M(P)$.
These properties are the basis of FIM, either for candidate generation or pattern growth methods, with horizontal or vertical data formats.

\section{Pattern-based biclustering}

The homogeneity criteria (Definition 1) in pattern-based approaches for biclustering is obtained through support and confidence-correlation metrics. Pattern-based approaches allow for an efficient and exhaustive space search that produces an arbitrary high number of biclusters within a flexible structure.

Definition 7. Given a matrix $A$ and a minimum support threshold $\theta$, a set of biclusters $\cup_{k} B_{k}$, where $B_{k}=\left(I_{k}, J_{k}\right)$, can be derived from the set of frequent itemsets $\cup_{k} P_{k}$ by either mapping $\left(I_{k}, J_{k}\right)=\left(\Phi_{P_{k}}, P_{k}\right)$ to compose biclusters with coherency on rows, or by mapping $\left(I_{k}, J_{k}\right)=\left(P_{k}, \Phi_{P_{k}}\right)$ to compose biclusters with coherency on columns.

A pattern-based approach to biclustering relies on an itemization step, where the original matrix is transformed into an itemset database, followed by the application of FIM methods under a low support threshold. For realvalue matrices, normalization and discretization procedures are applied. Then, the discrete value of each cell is concatenated with the respective column index. Each transaction of the target itemset database corresponds to a row with these new values. FIM is applied over the database to mine frequent patterns, which are then used to derive biclusters with constant values on rows. Constant values on columns can be mined using the transpose matrix. To find a more constrained type of biclusters, such as constant values overall, each item needs to be mined separately. Figure 2 illustrates how to deliver such types of biclusters using frequent patterns.

Although the state-of-the-art pattern-based biclustering methods follow this general behavior, they have varying structural specificities that affect both the efficiency 


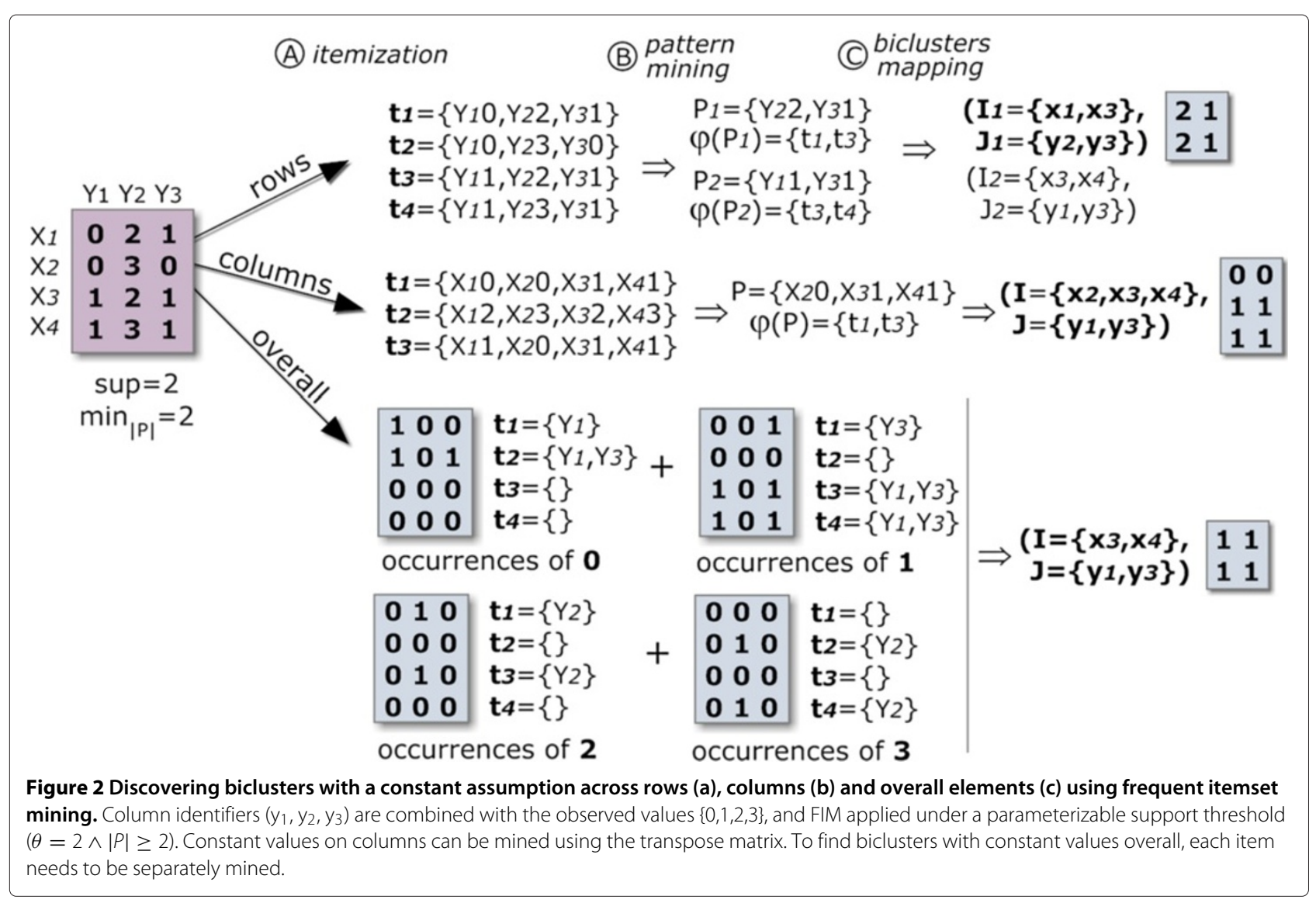

and the quality of the output. Two classes of PM-based biclustering approaches can be considered: approaches that directly apply pattern miners over discrete matrices, and approaches that target numeric matrices by customizing the support metric. To our knowledge, BiModule [13], DeBi [10], Bellay's et al. [30] and GenMiner [15] are the state-of-the-art methods for this first class of PM-based biclustering. BiModule [11,13] allows for a parameterized multi-value itemization of the input matrix to discover constant biclusters derived from (closed) frequent patterns using the LCM miner [31]. DeBi [10] derives biclusters from (maximal) frequent patterns mined over binarized matrices using the MAFIA miner [32], and places key post-processing principles to adjust biclusters in order to guarantee their statistical significance. Bellay's et al. [30] use the Apriori miner with additional principles to evaluate the functional coherency of the discovered biclusters against the background noise. GenMiner [15] includes external knowledge within the input matrix to derive biclusters from association rules that relate annotations (external grouping of rows or columns) with computed clusters of rows and columns from (closed) frequent patterns using CLOSE [33]. Although other biclustering approaches seize contributions from these previous works $[34,35]$, we do not refer to them as PMbased appproaches if the core mining task does not rely on FIM.

The itemization step is optional for the second class of methods [36]. To our knowledge, RAP [14], RCB discovery [36] and ET-bicluster [37] are the state-of-the-art methods in this context. RAP [14] plugs an adapted rangebased metric to mine constant biclusters on rows (or columns), while RCB discovery targets biclusters with constant values overall [36]. ET-bicluster extends the previous approaches to discover noisy biclusters, although an exhaustive enumeration of biclusters is not guaranteed [37]. Alternative support metrics with dedicated Apriori-based searches have been additionally referred in literature [38-40].

\section{BicPAM: pattern-based biclustering}

The proposed pattern-based biclustering approaches (BicPAM) are an ordered composition of the three-stage: mapping, mining (pattern discovery), and closing (or postprocessing) steps. BicPAM relies on both existing and novel principles for each step. The core step is the mining step, corresponding to the application of the target pattern miners. This step is driven by the considered pattern 
discovery approach, target patterns and search properties. The mapping step consists in the itemization of a realvalue matrix into an itemset matrix. This step is driven by normalization and discretization criteria and it may use different principles to handle outlier, numeric or missing elements. Finally, the closing step consists on the postprocessing of the output patterns to affect the structure and quality of the target biclusters. Figure 3 clarifies how BicPAM relies on the existing pattern-based contributions and pinpoints the novel principles proposed for each step.

The homogeneity criteria can be intentionally defined to search for specific types and structures of biclusters and to affect their quality. The bicluster type depends on the allowed coherency patterns and on their orientation (row, column or overall), the solution structure depends on the number, size and positioning of biclusters, and, finally, the quality defines the allowed noise associated with a single bicluster or with a set of biclusters.

BicPAM is introduced in three following sections. First, we describe the core steps of BicPAM (BicPAM outline). Second, we go further and incorporate new methods to deal with missing values and arbitrary high input levels of noise (Affecting the quality of pattern-based biclusters). Finally, we propose further algorithmic solutions for the discovery of biclusters allowing symmetries and following additive and multiplicative assumptions (Allowing more flexible types of biclusters).

\section{BicPAM outline}

This section describes the structural behavior of BicPAM by surveying principles for the mining, mapping and closing steps. These principles are either derived from the existing pattern-based approaches for biclustering or from advances in the pattern mining field.

\section{Mining step}

Understandably, non-constrained settings, where the number of biclusters and their properties is not known apriori, require efficient searches. Pattern mining approaches have been tuned during the last decades to be computationally efficient. Therefore, their adequate use for biclustering is critical and depends essentially on three points described below: 1) the adopted pattern-based approach to biclustering, 2) the target pattern representation, and 3) the search strategy.

\section{1) Pattern-based Approach}

Definition 8. Let $\mathcal{L}$ be a set of ordinal items, a bicluster is a sub-matrix $(I, J) \subseteq A$ with its elements $a_{i j} \in \mathcal{L}$ defining a pattern profile. A constant bicluster can follow: i) an overall constant assumption where $a_{i j}=c$ and $c \in \mathcal{L}$, ii) a column-based constant assumption where $a_{i j}=c_{j}$ and $c_{j} \in \mathcal{L}$, or iii) a row-based constant assumption where $a_{i j}=c_{i}$ and $c_{i} \in \mathcal{L}$.

Pattern-based biclustering under a constant assumption is the ordinary case. DeBi [10], BiModule [13] or GenMiner [15] only target this type of biclusters. These approaches either rely on Frequent Itemset Mining (FIM) or on association rules, which contrasts with traditional approaches $[9,18]$. The support threshold defines the minimum number of rows in a bicluster. In the context of gene expression, a low support is critical since high expression coherency is only observed for small groups of genes and

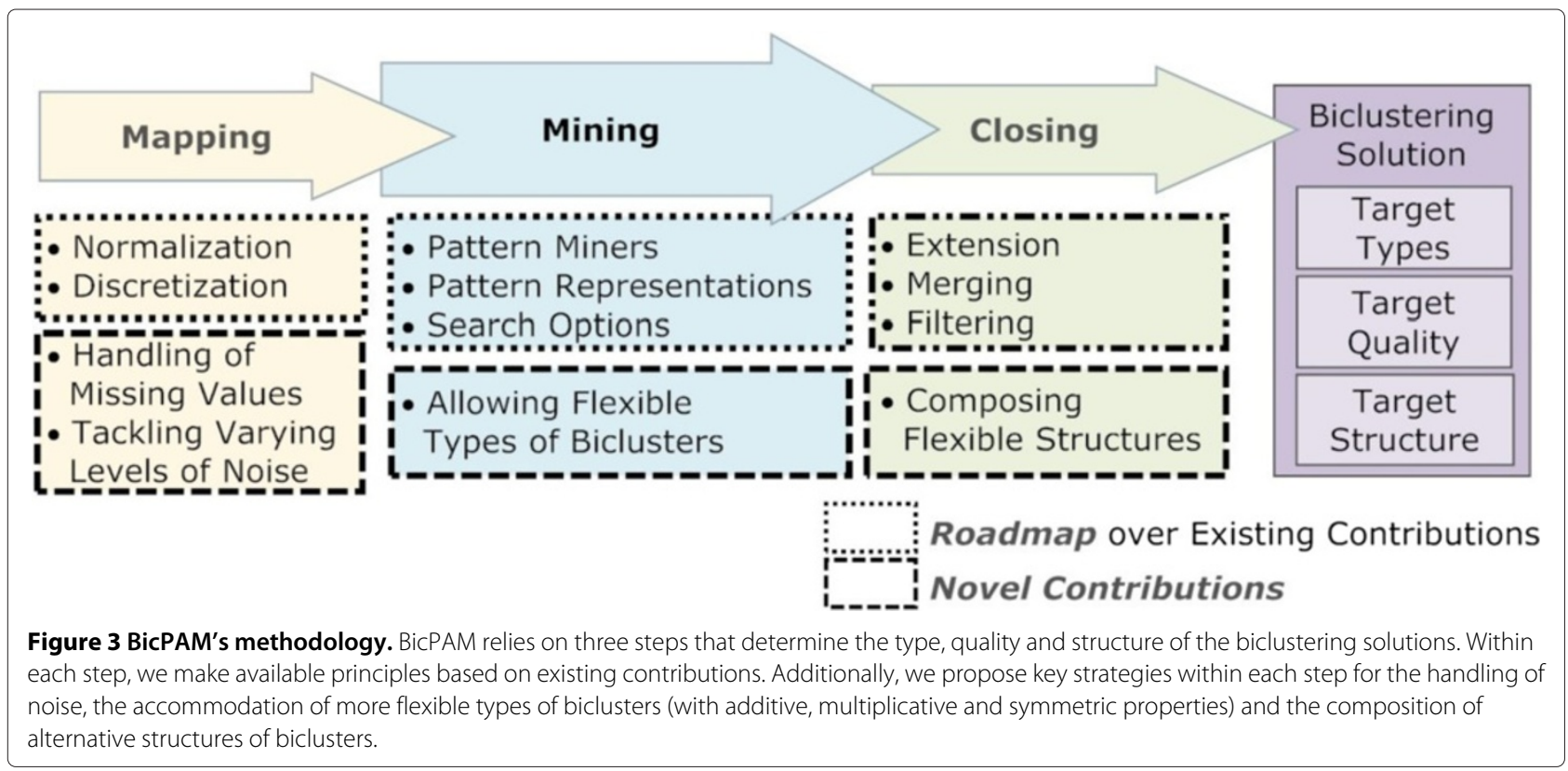


conditions. Additionally, a post-pruning to the frequent itemsets can be performed in order to filter frequent itemsets below a minimum number of columns and above a maximum number of rows and columns.

From the point of view of an itemized database, the FIMbased biclusters are perfect biclusters, that is, they do not allow value-variations in any of its elements. Contrasting, from the point of view of the input real-value matrix, these biclusters can handle noise since two elements with the same item may be numerically distant. The number of items can be used to control the noise-tolerance. However, regardless of the number of items, a common drawback appears when two elements have similar real-values but different items assigned. We refer to this drawback as the items-boundary problem.

BiModule [11] and DeBi [10] are representative FIMbased biclustering approaches. Since their running time is comparable to greedy algorithms, they offer a simplistic way to deal with noise and overlapping structures [13]. However, the items-boundary problem can lead to the partitioning of large biclusters into smaller ones (with many being filtered as they no longer satisfy the support criterion).

In order to mine frequent itemsets with different properties, the notion of support of an itemset can be redefined. RAP [14] uses a customized anti-monotonic range support merit function. A FIM-based algorithm is used to discover range patterns from real-value matrices without the need for discretization. However, efficiency is strongly penalized.

An additional option to pattern-based biclustering is to derive biclusters from association rules. An association rule, an implication between two itemsets, can affect the properties of the corresponding bicluster as it constrains the levels of confidence among rows. Optionally, correlation metrics can be adopted to augment the confidence-support metrics with new interestingness criteria. GenMiner [15] uses association rules to compose biclusters. However, the adoption of association rules is only preferred over FIM-based approaches when knowledge regarding the dependencies across rows (or columns) is available.

BicPAM uses frequent itemsets as the default patternbased option to biclustering. Range-based approaches are only selected for small-to-medium datasets. Finally, in the presence of domain knowledge (such as functional groups of genes or dependencies on conditions), BicPAM relies on association rules to compose biclustering solutions.

\section{2) Pattern Representations}

The target pattern representation depends essentially on: 1) the selected type and structure of biclusters, and
2) the post-processing needs. Efficiency is not a strong criterion, since only subtle gains are observed for methods that target constrained representations, such as closed and maximal representations.

The use of all frequent itemsets leads to biclustering solutions with a high number of (potentially redundant) biclusters (if contained by another bicluster), which can degrade the performance of the mining and closing steps. Contrasting, the use of maximal itemsets leads to biclusters with the columns' size maximized. Maximal itemsets for biclustering are adopted in DeBi [10]. Such flattened biclusters are particularly interesting when there is an extension step to be performed to include new rows for the discovered biclusters. However, since both vertical and smaller biclusters are avoided, maximal-based biclusters lead to incomplete solutions as they are just a subset of all valid biclusters.

Finally, by using closed itemsets, we allow for overlapping biclusters only if a reduction on the number of columns from a specific bicluster results in a higher number of rows. Note that to obtain maximal biclusters biclusters that cannot be extended without the need of removing rows and columns - closed patterns need to be used instead of maximal patterns. FIM-based BiModule [13] and rule-based GenMiner [15] use closed itemsets as the means to compose biclusters.

BicPAM uses frequent closed patterns as the default representation. The set of all and maximal frequent patterns are also made available in BicPAM. An illustration on how different types of pattern representations lead to structurally different biclustering solutions is provided in Figure 4.

\section{3) Search Strategies}

The definition of the search setting depends essentially on: 1) the fit of the search with the target biclustering task, and 2) the chosen implementation.

The choice on whether to use Apriori-based [41], pattern-growth [42] or combined approaches [43], mainly depends on the dataset density and fixed support thresholds. Dense matrices under low support thresholds benefit from pattern-growth or combined methods. The choice on whether to use a mining method that has a vertical versus an horizontal data format [43] depends essentially on the type of biclusters we are targeting. If we want to find constant values across rows or on both dimensions, we usually benefit from using searches over horizontal data formats [35]. This is particularly true for most GE matrices where the total number of genes largely exceeds the total number of conditions. If we want to find constant values across columns (when $n>m$ ), a vertical data format should be the choice, as the performance searches based horizontal formats 


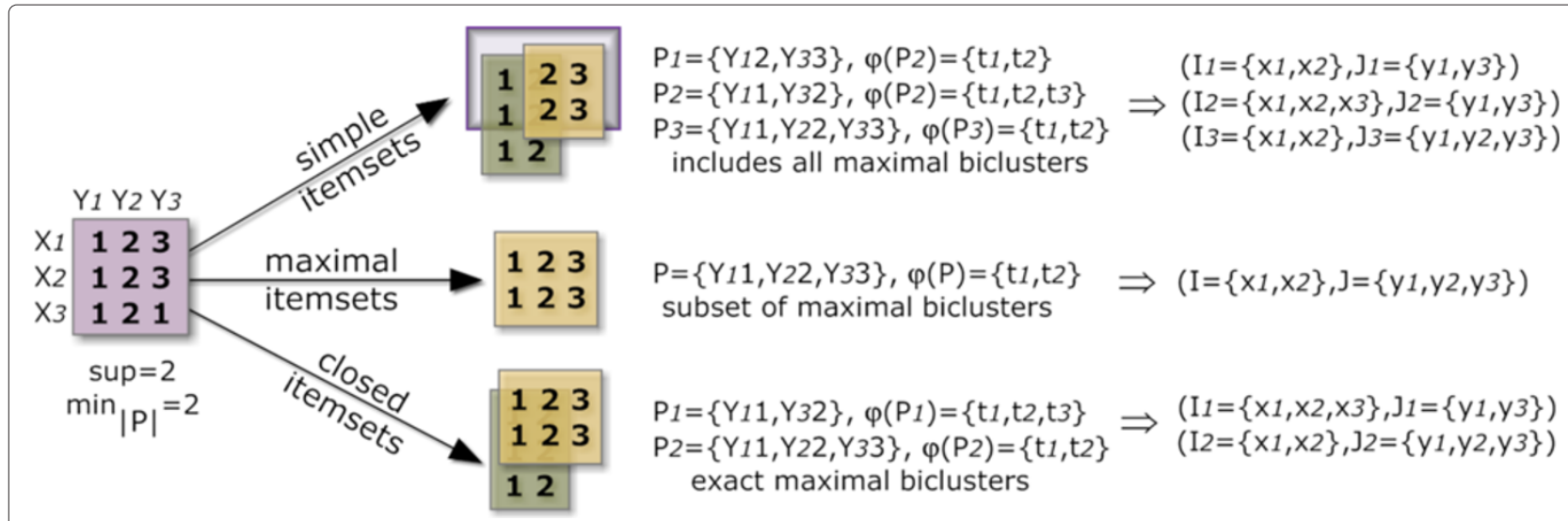

Figure 4 Comparison of biclustering solutions using frequent itemsets, maximal frequent itemsets and closed frequent itemsets.

exponentially degrades with an increasing number of items.

Several algorithms were developed for each of these search strategies. However, their properties should be carefully assessed, as their nature is most of the times optimized towards specific sets of datasets. In the DeBi [10], BiModule [11] and GenMiner [15] biclustering tasks, Mafia [32], LCM [31] and CLOSE [33] are, respectively, the algorithmic choices.

BicPAM makes available a variant of FP-Growth that traces the set of transactions per frequent pattern [44] (default option), Charm [45], AprioriTID [41] and Eclat [43]. Finally, Carpenter [46] and Cobbler [47] are additional algorithmic choices in BicPAM to compose biclusters with a large number of columns and for large-scale datasets.

\section{Mapping step}

Normalization techniques are often required to enhance differences across rows and/or columns. Alternative methods have been reported [34,48]. BicPAM allows the normalization criteria to be applied in the context of a row, a column or the overall matrix. Additionally, it makes available a zero-mean value to allow for symmetries and to provide a simple setting for the approximation of probabilistic distributions. In the presence of missing and outlier elements, a masking bitmap can be used in order to exclude them from the computation of the mean and dispersion metrics.

Discretization is an additional key step for patternbased methods relying on itemset databases. Although discretization may imply loss of information, it alleviates the noise dilemma [26] and it is the cost to pay for exhaustive searches. BicPAM makes available multiple discretization options with key implications on the target solution. Two axes are considered: 1) the number of items (also referred as symbols) and 2) the target method to map the normalized real-value matrix into a itemset database. Increasing the number of items is commonly used to improve quality, but it reduces the average size of biclusters and the number of biclusters produced. A sensitivity analysis on the impact of choosing different number of items was performed in Bidens [34] and BiModule [13].

The three discretization methods made available in BicPAM are illustrated in Figure 5. The use of fixed ranges (potentially equal sized intervals between the observed maximum and minimum) is the simplest discretization option, but commonly leads to an accentuated weak distribution of items and is prone to the items-boundary problem. The first problem can be corrected using a percentage-based method for the depth partitioning of items that leads to intervals containing approximately the same number of items. Bidens [34] uses this equal-depth partitioning method over a data context where outliers are temporarily removed. Finally, alternative distributions can be used to combine the properties of the previous solutions, such as the setting proposed in Nordi [15]. By finding multiple suitable curves (for each row or column) or one suitable overall curve for approximating the matrix, we can either use threshold methods or directly compute the statistical cutoff points to create equallydistributed areas. In the illustration, a Gaussian distribution is selected to minimize the loss of potentially relevant biclusters.

\section{Closing step}

Similarly to mining and mapping options, post-processing criteria can be used to minimize the two challenges of the noise dilemma. One challenge results from a too restrictive noise tolerance, commonly associated with considering a high number of items, which leads to many small sized biclusters. The other challenge is related with heightened levels of noise allowance, commonly appearing in binarized partitions and under a relaxed levels of support or confidence. To handle these challenges and to treat the problem of the explosion of valid biclusters 


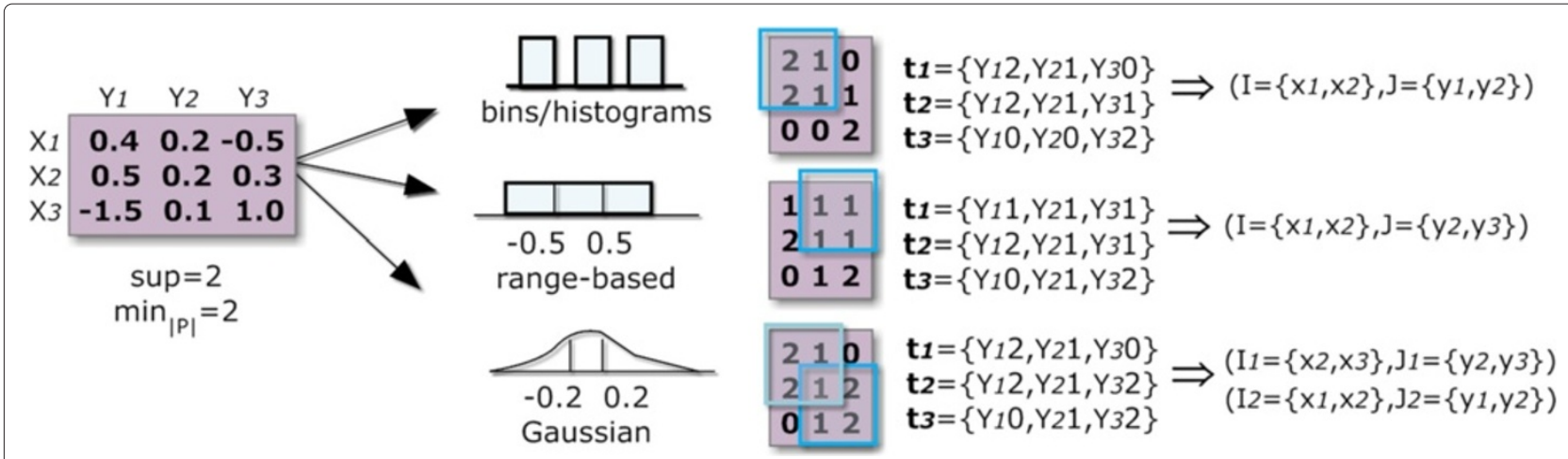

Figure 5 Impact of discretization options available in BicPAM.

(commonly connected with overlapping biclusters), BicPAM enables the use of criteria structured according to three stages: 1) extension, 2) merging and 3) filtering.

\section{1) Extension Options}

Three optional and non-exclusive strategies can be used to extend the discovered biclusters such that the resulting solution still satisfies some pre-defined homogeneity criteria. First strategy consists on the use of statistical tests to include rows or columns over each bicluster as proposed in DeBi [10]. Second strategy relies on traditional approaches and on their merit functions for further extensions as long as the solution satisfies either the intraor inter-bicluster homogeneity criteria. Finally, we propose a third strategy that uses patterns discovered under more relaxed criteria (such as lower support-confidence thresholds) to guide the extension step. When considering lower supports, new columns and rows can be added to the original frequent patterns. Similarly, more relaxed association rules, with less restrictive ways to group the antecedent-consequent, can be used to guide the extension step. The use of simple thresholds, of statistical tests or of merit functions to verify if the bicluster is valid can either be computed using the discretized matrix (item matchings) or, more interestingly, the distances from the original real-value matrix.

\section{2) Merging Options}

Merging operations serve two goals: noise allowance and overall biclustering structure manipulation. The first goal is driven by the observation that when two biclusters share a significant area it is probable that their merging composes a larger bicluster still respecting some homogeneity criteria. Commonly, such decomposition is related with the items-boundary problem or with a missing value. The simplest criterion to allow the merging is either to rely on the overlapping area (as a percentage of the smaller bicluster), to compute the overall noisy percentage after the merging, or to use advanced homogeneity criteria (potentially relying on the real-values provided by the input matrix). State-of-the-art procedures to efficiently merge pattern-based biclusters include $[49,50]$.

\section{3) Filtering Options}

Filtering is possible at two levels: 1) at the bicluster level, and 2) at the row-column level. The first type of filtering is required to remove duplicates and biclusters that are contained in larger biclusters. The existence of biclusters included in larger biclusters is a necessary result of the extension-merging options and it is a common problem when adopting mining approaches that do not rely on condensed pattern representations. Both DeBi [10] and BiModule [13] provide alternative heuristics to efficiently perform this type of filtering.

The second type of filtering can be used to exclude rows or columns from a particular bicluster in order to intensify its homogeneity. This is usually the case when a low number of items is considered, leading to highly noisetolerant biclusters. For this purpose, similarly to extension options, we can rely on three strategies: 1) use statistical tests on each row and column of a particular bicluster in order to identify removals, 2) rely on existing greedyiterative approaches and maximize their merit functions (which can imply a reduction on the size of biclusters), and 3) discover patterns under more restrictive conditions (as higher support and confidence thresholds).

\section{Affecting the quality of pattern-based biclusters}

BicPAM options with impact on the solution quality include:

- Mining step options, including the approach, the support-confidence thresholds, and the pattern representations;

- Mapping step options, including the number of items and the normalization-discretization techniques;

- Closing step options, including the selected extension, merging and filtering approaches, and 
their criteria thresholds (percentage of noise, overlapping degree, statistical significance levels).

Below, we describe new strategies that BicPAM makes available to handle varying levels of missing values and input noise, and to compose multiple structures of biclusters.

\section{Handling missing values}

The input matrices can have an arbitrary high number of missing values, as it is common in GE matrices. A nontreated missing value may result in the loss of a critical row and of a column across one or more biclusters. Three different strategies can be applied to treat missing values: 1) removal, 2) replacement, and 3) handling as a special value. The simplest method is to remove the containing row or column (usually the dimension with smaller size).

Many hole-replacing methods have been proposed [51-53], alleviating the referred problem, although introducing additional noise that can significantly decrease the homogeneity of the output biclusters. For this reason, we propose the use of an additional item that is specially handled according to a level of relaxation handled by the user, as illustrated in Figure 6. The lowest constrained setting (relaxed) replaces the missing item by all other adopted items, which again results in transactions with varying size. The medium constrained setting ( $\delta$-replace) considers multiple items around its value-estimation. If the difference between the estimated value and the centroidvalue of a discretization range is less than $\delta$, then the item assigned to the range is added. In BicPAM, the default imputation method is based on the mean values for the four nearest neighbor rows. BicPAM default $\delta$ distances guarantee a lower bound of two items and an upper bound of three items. The highest constrained setting (restrictive) removes missing items.

\section{Handling varying levels of noise}

A key direction to pattern-based biclustering is to consider multiple levels of noise by following one of the three strategies illustrated in Figure 7. First strategy (reduced number of items) hierarchically joins contiguous items (items are viewed as being ordinal and no longer nominal) to mine biclusters over matrices with different number of items. Optimizations to this strategy can be made by collapsing items only for some critical areas of the matrix where the presence of biclusters is scarcer. Understandably, the level of noise should be maintained by each bicluster, so that closing steps can be adapted in respect to the quality of the target bicluster. Second strategy (relaxedto-restricted extensions) considers varying levels of noise only after the mining. For instance, the merging of constant biclusters can follow a statistical test sensitive to the closeness of different items (heuristics based on overlapping rows-and-columns should also be considered). Third strategy (multiple items) associates one or more items to each element based on a parameterized threshold. Different criteria can be defined to assign a varying number of items per element $a_{i j}$. Each element can be mapped into two-to-three items based on the distance to their centroids leading to transactions with multiple sizes.

\section{Producing alternative biclustering structures}

Since the number of biclusters is neither fixed nor depends on the satisfaction of local coverage criterion, pattern-based approaches provide a heightened flexibility for the composition of different biclustering structures. A pattern-based solution is non-exhaustive, non-exclusive and allows overlaps. The task of composing different structures has been poorly addressed in literature, and rather seen as the byproduct of biclustering methods [1]. Below, we introduce a set of principles to compose multiple structures made available in BicPAM.

For an exhaustive structure (either overall, across rows or across columns), biclusters can be incrementally

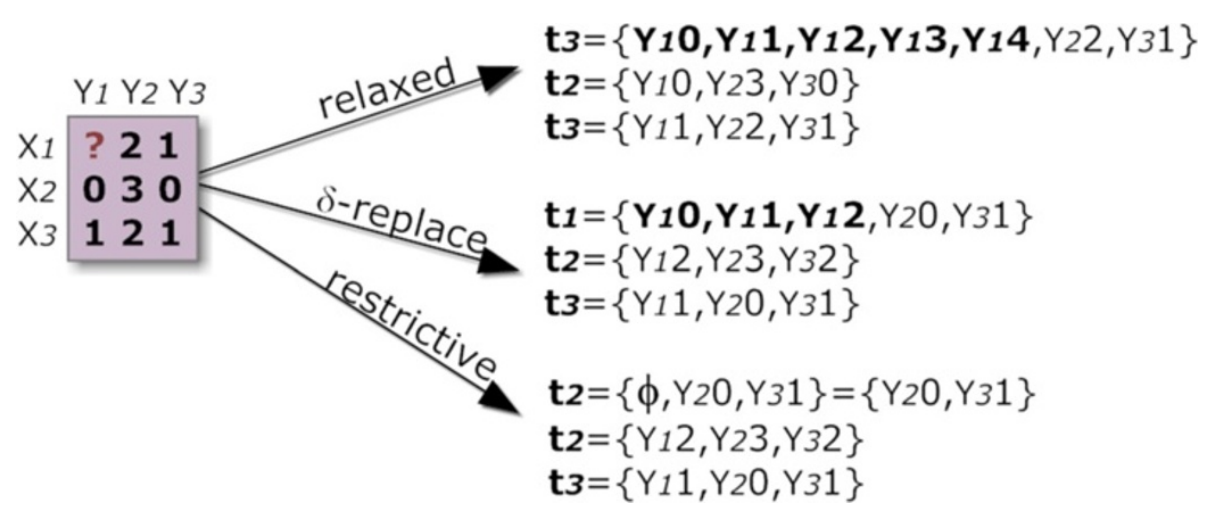

Figure 6 Mapping methods to handle missings: relaxed, conservative ( $\delta$-replace) and restrictive alternatives to imputation. 


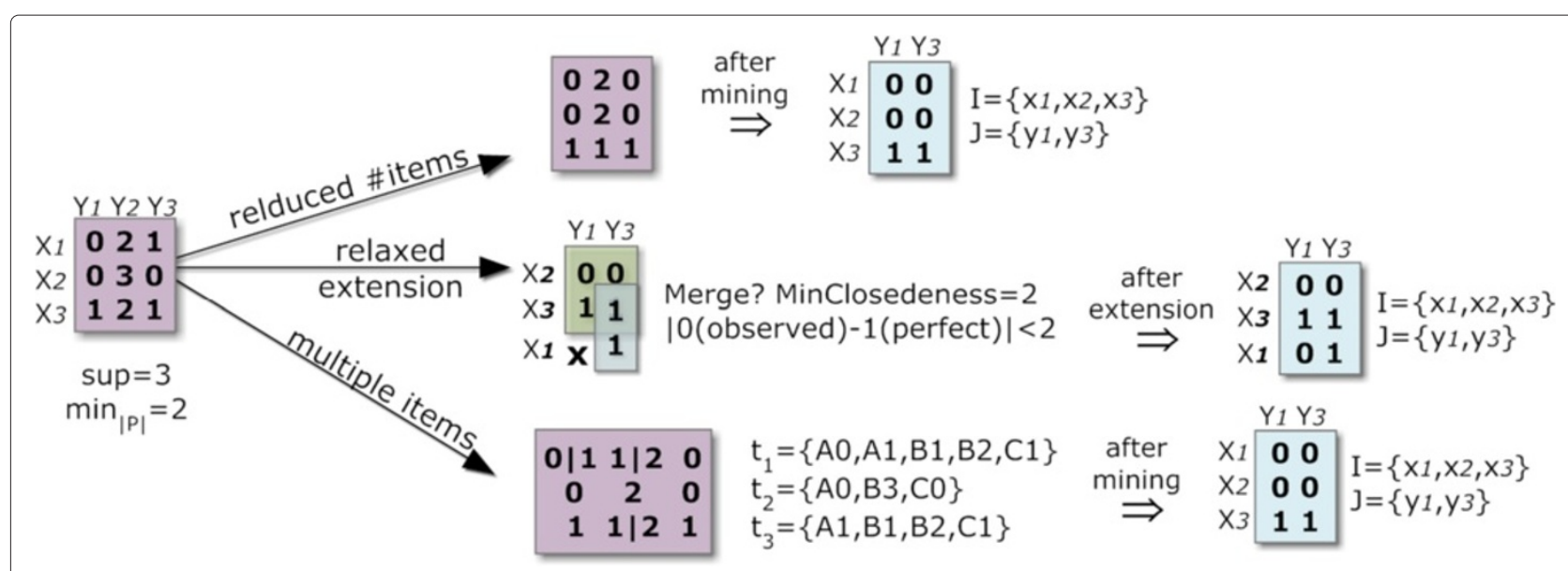

Figure 7 Strategies to deal with noise-relaxations.

merged following, for instance, an hierarchical criteria based on the proximity and the area of biclusters, until all the matrix is covered. If the goal is an exclusive structure (either overall, across rows or across columns), a simple strategy is to merge biclusters in order to reduce overlapping across one or both dimensions and, additionally, to filter biclusters that share rows or columns following an relevance criterion (as size or noise level) until exclusivity is guaranteed. Closing options can be specifically used to produce other alternative structures with sharp usability (no need to change the core tasks of pattern-based approaches).

\section{Allowing more flexible types of biclusters}

Below, we extend BicPAM to consider more flexible expression patterns: additive, multiplicative and symmetric coherency.

\section{Coherency under additive-multiplicative assumption}

Definition 9. A bicluster $(I, J)$ follows an additive model if $a_{i j}=c+\alpha_{i}+\beta_{j}+\eta_{i j}$, where $c$ is the typical value within the bicluster, $\alpha_{i}$ is the adjustment for row $i \in I, \beta_{j}$ is the adjustment for column $j \in J$ and $\eta_{i j}$ is the noise associated with the element. A bicluster $(I, J)$ follows a multiplicative model if $a_{i j}=c^{\prime} \times \alpha_{i}^{\prime} \times \beta_{j}^{\prime}+\eta_{i j}$, which is equivalent to the additive model when $c=\log c^{\prime}, \alpha_{i}=\log \alpha_{i}^{\prime}$ and $\beta_{j}=\log \beta_{j}^{\prime}$.

We propose two pattern-based strategies for the discovery of biclusters with non-constant models of coherency. The first strategy is to use local normalization procedures to correct row- or column-based differences and then to map the task into the search for constant biclusters.

The second strategy, the default BicPAM option, is to iteratively perform alignments on each column (or row). This guarantees that all the alignments needed to compose these biclusters are considered. Therefore, the selected pattern miner is applied either $m$ (or $n$ ) times, leading to a higher computational complexity. Figure 8 illustrates this strategy.

An additive alignment over a target column $y_{j}$ can be computed by adding for each element on the row $x_{i}$ the difference between the maximum of the column and the discretized value $\max \left(y_{j}\right)-a_{i j}$. A multiplicative alignment over a target column $y_{j}$ can be computed by adding, for each element on the row $x_{i}$, the least common multiple between the maximum of the column and the discretized value $\operatorname{lcm}\left(\max \left(y_{j}\right), a_{i j}\right)$. The resulting number of items under an additive assumption is in the worst case the double of the number of items initially considered. The number of final items under a multiplicative model is the size of the $l \mathrm{~cm}$ combinations across the initial items. As illustrated in Figure 8, a distance-based $\delta$-error can be considered to gather close items in the multiplicative model due to the lower probability of finding coherent biclusters as a consequence of the resulting large number of items.

\section{Coherency under symmetrical assumption}

A critical, but less studied, type of biclusters is biclusters with coherent values under symmetrical assumption, also referred as biclusters with sign-changes in literature [1]. Two rows or columns from a bicluster allowing symmetries may have similar absolute values differing in sign. Such biclusters can simultaneously capture activation and repression mechanisms within a biological process.

Definition 10. A bicluster $(I, J)$ following a symmetric model has either: $i$ ) symmetries on rows $\hat{a}_{i j}=c_{i} \times a_{i j}$, where $c_{i} \in\{-1,1\}$ is the symmetry factor for each row of the bicluster and $a_{i j} \in \mathbb{R}$ is a bicluster element defined according to a constant, additive or multiplicative model, or ii) on columns $\hat{a}_{i j}=c_{j} \times a_{i j}$, where $c_{j} \in\{-1,1\}$ is the column 


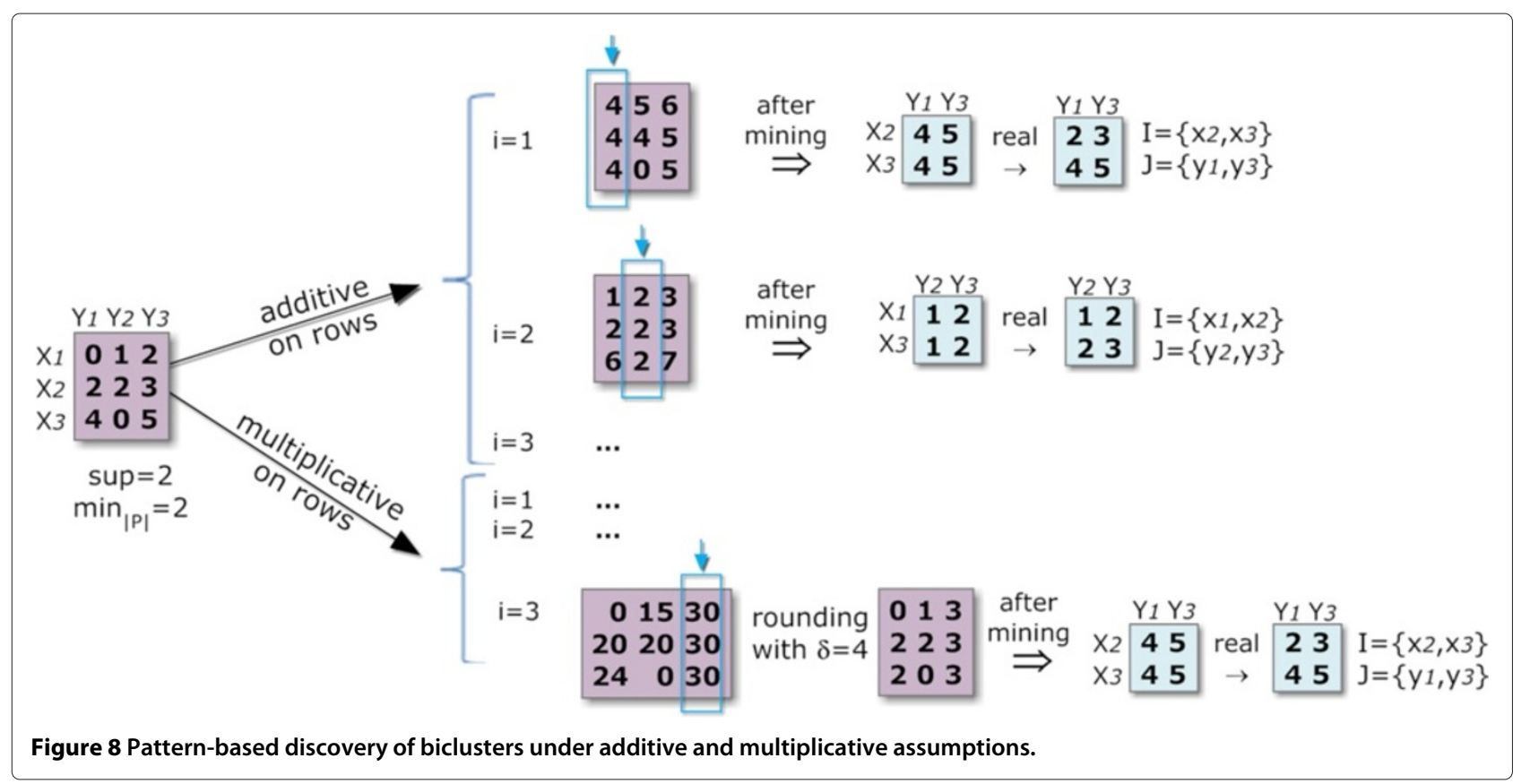

symmetry factor and $a_{i j} \in \mathbb{R}$ is an element of a bicluster with coherent values.

For the purpose of finding biclusters with symmetries, the normalization should satisfy the zero-mean criterion. Additionally, if the number of considered items is odd, there is one item being its own symmetric that must be specially handled.

One option is to align the sign of activity of each row (or column) in order to guarantee consistency of signals for a target column (or row). The top example in Figure 9 illustrates this strategy. An iterative mapping for every column (or row) is possible, although additional efficiency can be achieved by stopping the search when all the sign combinations have been achieved. Nevertheless, the worst case requires the application of a pattern miner $m$ times (or $n$ times). Note that filtering is a critical step needed to remove potential duplicates resulting from repetitions of alignments for subsets of rows (or columns).

The combination of this strategy with the search for biclusters under an additive or multiplicative model can be expensive ( $m \times m$ times iterations). Therefore, BicPAM makes available an additional option to combine the use of the sign and of the additive or multiplicative adjustments together for every column (or row). This model (combined sign and coherent model) is not equivalent to the previous

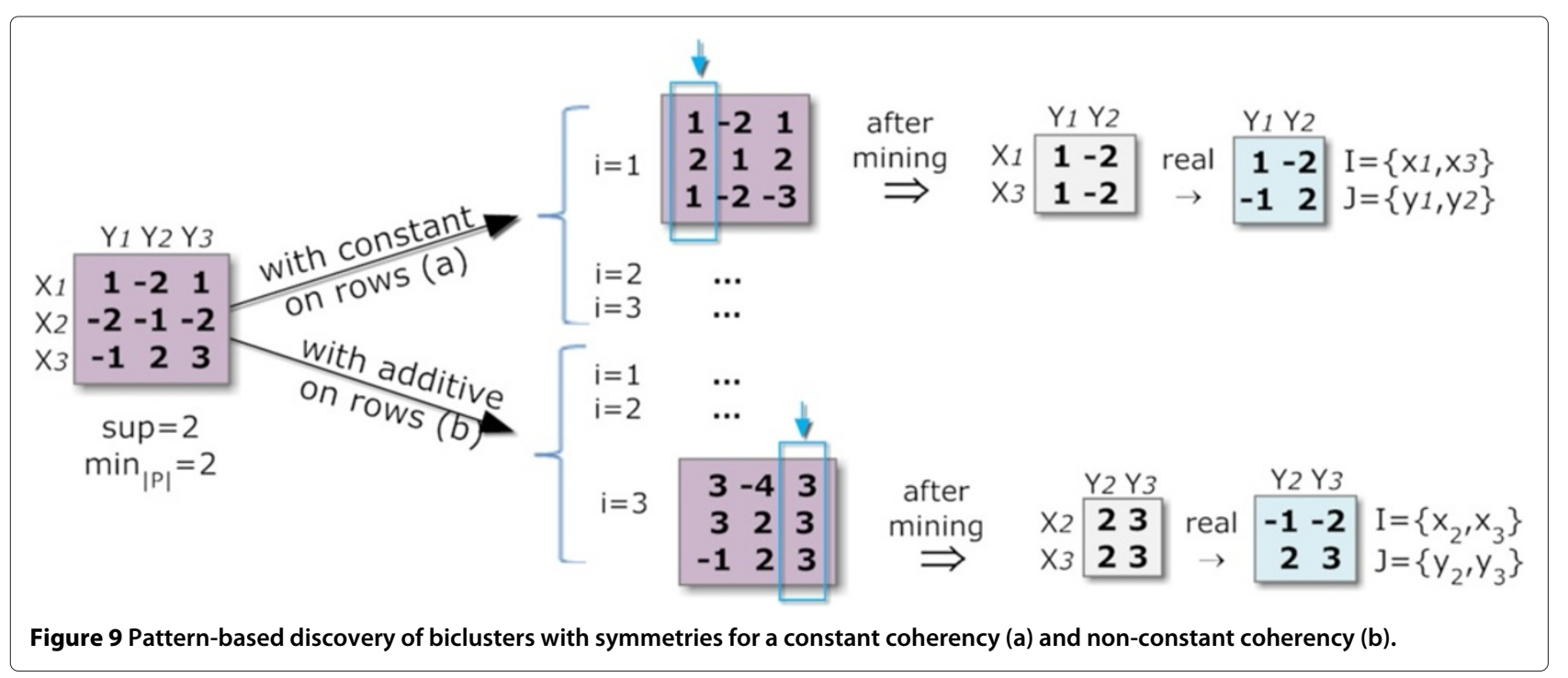


model (sign plus coherent model), since it assumes that additions or multiplications are not absolute but depend on the activity slope sign. Here, the value adjustment of a particular element is also affected by the sign, which can lead to an additional number of items. This strategy is illustrated in the bottom example of Figure 9.

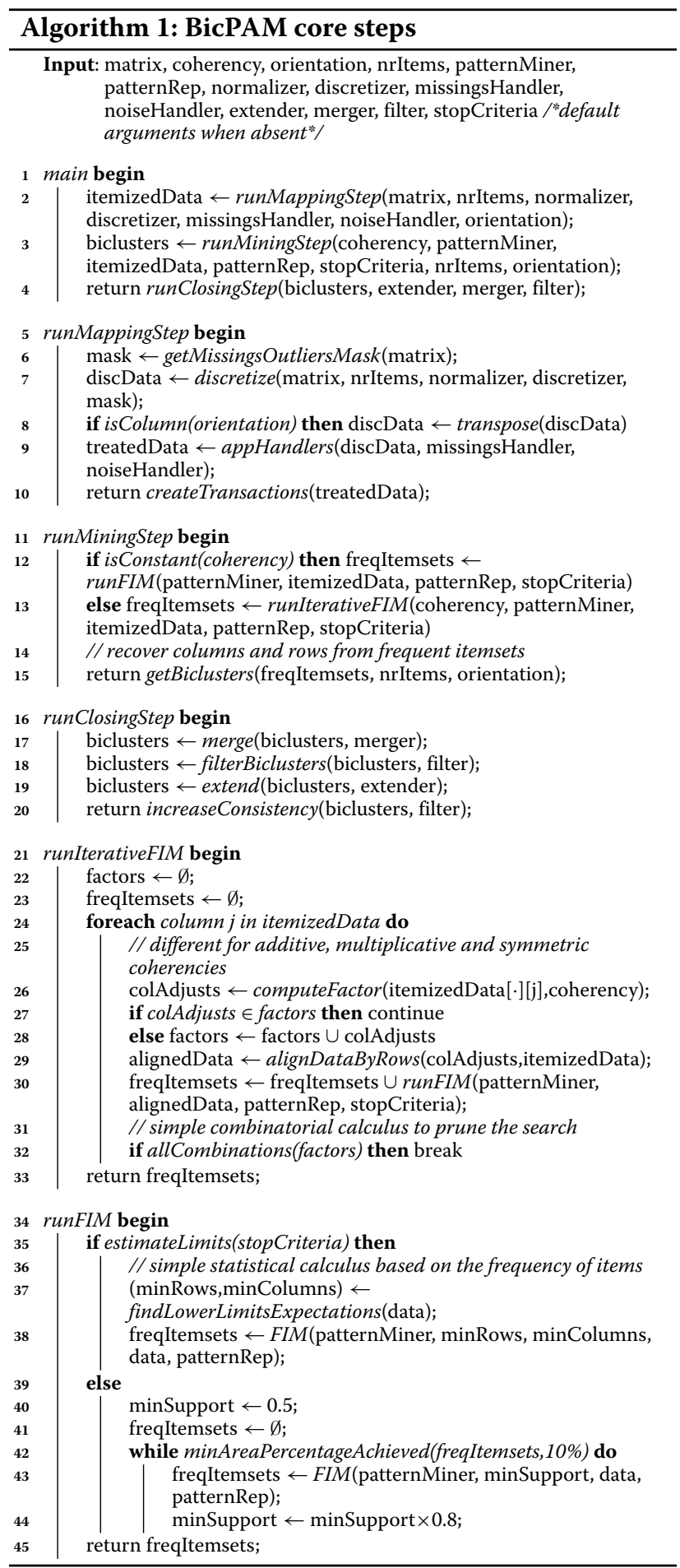

\section{BicPAM algorithm and complexity analysis}

The algorithmic basis of BicPAM is described in Algorithm 1. Although BicPAM follows a plug-andplay style, default and data-driven parameterizations are made available. In particular, lines 40-44 and 37 describe BicPAM behavior in the absence of user-driven parameterizations. This is performed by either relying on estimation procedures or on convergence criteria based on thresholds such as the relative area covered by biclusters or the minimum number of biclusters.

The computational complexity of BicPAM is bounded by the pattern mining task and computation of similarities among biclusters. For this analysis, we cover major computational bottlenecks related with each one of the three major steps of BicPAM. Within the mapping step: outlier detection, normalization, discretization, and noise correction procedures (such as the assignment of multiple items) are linear on the size of the matrix, $\Theta(n m)$. The optional distribution fitting tests and parameter estimations to dynamically select an adequate discretization procedure are also $\Theta(n m)$. These tests and estimations rely on the calculation of approximated statistical ratios [54]. Handling missings by removing the respective element or by replacing them by a special dedicated item is also $\Theta(n m)$. However, when an imputation method is selected, the complexity is upper bounded by $\Theta(h n m)$, where $h$ is the number of missing values. In BicPAM implementation, the nearest neighbor rows and columns are computed for the estimation of each missing value.

The cost of the mining step depends on two factors: the complexity of the pattern miner and the need for iterations for the discovery of non-constant profiles. The cost of the pattern mining task depends essentially on: the number and size of transactions $(\gamma \mathrm{nm}$, where $\gamma \geq$ 1 captures the increase in size related with noise and missings handlers), the frequency distribution of items $(\{\mathcal{L} \times Y\} \rightarrow \mathbb{N})$, the minimum support $\theta$, the pattern representation and the selected mining procedure. A detailed analysis of this complexity has been attempted in literature [55] and it is out of the scope of this paper. The reader should also keep in mind that there has been proposals to guarantee the scalability of pattern miners recurring to partitioning and approximation methods [12]. Let $\Theta(\wp(\gamma, n, m,|\mathcal{L}|, \theta))$, or simply $\Theta(\wp)$, to be the complexity of a pattern mining task. When there is the need for the iterative application of the core mining procedure, the overall search is bounded by $\Theta(d \times$ $\wp)$, where $d=\min \left(\left(\begin{array}{l}n \\ 2\end{array}\right), m\right)$ when allowing symmetries, $d=\min \left(\left(\begin{array}{c}n \\ |\mathcal{L}|\end{array}\right), m\right)$ when allowing shifts, and $d=$ $\min \left(\left(\begin{array}{c}n \\ \sharp c m\end{array}\right), m\right)$ when allowing scaling factors.

The cost of the closing step depends essentially on two factors: the complexity of computing similarities among biclusters (required for merging and filtering biclusters) 
and the complexity of extending and reducing biclusters. To compute similarities a tree structure is created where each node represents a gene and each leaf corresponds to a bicluster. Only biclusters sharing a branch over a threshold based on the input overlapping degree are candidates for merging and filtering. Filtering a bicluster results in the removal of its leaf and dedicated nodes. Merging two biclusters results on the combination of the target branches. These tasks have an average complexity of $\Theta\left(\left(\begin{array}{c}k \\ k / 2\end{array}\right) \bar{r} \bar{s}\right)$, where $k$ is the number of biclusters and $\bar{r} \bar{s}$ their average size. Extending biclusters relies on quick tests based on the coherency of each new column or row and therefore the complexity of this task is respectively $\Theta\left(k^{\prime} \bar{r} m\right)$ or $\Theta\left(k^{\prime} n \bar{s}\right)$, where $k^{\prime}$ is the number of biclusters after merging and filtering. Removing rows or columns from biclusters is $\Theta\left(k^{\prime} \bar{r} \bar{s}\right)$.

In this context, the complexity of BicPAM is bounded by $\Theta\left(h n m+d \wp+\left(\begin{array}{c}k \\ k / 2\end{array}\right) \bar{r} \bar{s}+k^{\prime}(\bar{r} m+n \bar{s})\right)$, which for settings resulting in a large number of biclusters after the mining step $\left(k \gg k^{\prime}\right)$ is approximately $\Theta\left(d \wp+\left(\begin{array}{c}k \\ k / 2\end{array}\right) \bar{r} \bar{s}\right)$.

\section{Results}

In this section, we present an extensive experimental evaluation showing that BicPAM is effective and computationally efficient. BicPAM was implemented in Java (JVM version 1.6.0-24). The following experiments were run in an Intel Core i3 $1.80 \mathrm{GHz}$ with $6 \mathrm{~GB}$ of RAM.

The results were collected and analyzed in four steps. Section "Comparison of biclustering approaches in synthetic data" compares the performance of BicPAM against state-of-the-art biclustering approaches. In Section "Performance analysis in synthetic data", BicPAM's behavior is extensively assessed in synthetic datasets with varying size, noise, sparsity and background distributions. The biological relevance of BicPAM's results is analyzed in Section "Results in real data". Finally, Section "Comparison of pattern-based biclustering approaches" goes further on comparing BicPAM and its pattern-based peers. Below, we describe the evaluation metrics and data settings used.

Evaluation metrics. Biclustering solutions have been assessed using multiple evaluation criteria. In the presence of hidden/planted biclusters, $\mathcal{H}=\left\{H_{1}, . . H_{g}\right\}$, clustering metrics ${ }^{\mathrm{a}}$, match scores $[2,58]$ and relative nonintersecting area (RNAI) $[59,60]$ have been used. Match scores (MS) [58] assess the similarity of solutions based on the Jaccard index. $M S(\mathcal{B}, \mathcal{H})$ defines the extent to what found biclusters match with hidden biclusters, while $M S(\mathcal{H}, \mathcal{B})$ reflects how well hidden biclusters are recovered (1). RNIA [59] measures the overlap area between the hidden and found biclusters. To distinguish if several or few of the found biclusters cover a hidden bicluster, clustering error (CE) [60] is a critical extension. To take into account the number of biclusters in both sets, Hochreiter et al. [2] introduced a consensus score by computing similarities between all pairs of biclusters (2). We refer to this metric as FABIA Consensus (FC). Let $S_{1}$ and $S_{2}$ be, respectively, the larger and smaller set of biclusters from $\{\mathcal{B}, \mathcal{H}\}$, and $M P$ be the assigned pairs using the Munkres method based on overlapping areas [61], MC and FC are defined as:

$$
\operatorname{MS}(\mathcal{B}, \mathcal{H})=\frac{1}{|\mathcal{B}|} \Sigma_{\left(I_{1}, J_{1}\right) \in \mathcal{B}} \max _{\left(I_{2}, J_{2}\right) \in \mathcal{H}} \frac{\left|I_{1} \cap I_{2}\right|}{\left|I_{1} \cup I_{2}\right|}
$$

$$
\begin{aligned}
\mathbf{F C}(\mathcal{B}, \mathcal{H})= & \frac{1}{\left|\mathcal{S}_{1}\right|} \Sigma_{\left(\left(I_{1}, J_{1}\right) \in \mathcal{S}_{1},\left(I_{2}, J_{2}\right) \in \mathcal{S}_{2}\right) \in M P} \\
& \times \frac{\left|I_{1} \cap I_{2}\right| \times\left|I_{1} \cap J_{2}\right|}{\left|I_{1}\right| \times\left|J_{1}\right|+\left|I_{2}\right| \times\left|J_{2}\right|-\left|I_{1} \cap I_{2}\right| \times\left|J_{1} \cap J_{2}\right|} .
\end{aligned}
$$

In the absence of hidden biclusters, merit functions can be used as long as they are not biased towards the merit criteria used within the approaches under comparison. Examples include the commonly used mean squared residue (MSR) [62] and its extension [16], or the Pearson's correlation coefficent [59] sensitive to shifting-scaling properties. Finally, domain-specific evaluations can be used by computing statistical enrichment $p$-values in biological contexts $[10,63]$.

Data settings. Gene expression data and two sets of synthetic datasets were used to evaluate BicPAM performance. The first set corresponds to the datasets generated by Hochreiter et al. [2]. These datasets simulate specific characteristics of gene expression data, such as heavy tail properties, using three settings: multiplicative models and additive models under signals according to $N\left( \pm 2,0.5^{2}\right)$ and $N\left( \pm 4,0.5^{2}\right)$ distributions [64]. Each setting has 100 datasets with 1000 genes, 100 conditions and 10 planted biclusters.

A second set of synthetic datasets with varying size and planted biclusters with varying degrees of expression was generated in the context of this work [65] (settings described in Table 1). We varied the size of the matrices up to 4.000 rows and 400 columns, maintaining the proportion between rows and columns commonly observed in gene expression data. The number and shape of the planted biclusters were also varied. The properties of the generated matrices were carefully chosen in order to follow properties from similar studies $[10,13]$.

The generated matrices were parameterized according to pre-specified number of items $(|\mathcal{L}| \in\{5,10,20\})$ and to an inputed bicluster type assumption (constant, additive, multiplicative and/or symmetric). The number of rows and columns for each bicluster followed a Uniform distribution over the ranges presented in Table 1 . We allow for overlapping biclusters, which can difficult the recovery of the original planted biclusters. Finally, a noise factor was randomly added over the background values. This 
Table 1 Properties of the generated set of synthetic datasets

\begin{tabular}{|c|c|c|c|c|c|}
\hline Matrix size ( $\sharp$ rows $x \sharp$ cols) & $100 \times 30$ & $500 \times 60$ & $1000 \times 100$ & $2000 \times 200$ & $4000 \times 400$ \\
\hline Nr. of hidden biclusters & 3 & 5 & 10 & 15 & 20 \\
\hline Nr. columns in biclusters & {$[5,7]$} & {$[6,8]$} & {$[6,10]$} & {$[6,14]$} & {$[6,20]$} \\
\hline Nr. rows in biclusters & {$[10,20]$} & {$[15,30]$} & {$[20,40]$} & {$[40,70]$} & {$[60,100]$} \\
\hline Area of biclusters & $9.0 \%$ & $2.6 \%$ & $2.4 \%$ & $2.1 \%$ & $1.3 \%$ \\
\hline
\end{tabular}

noise factor is up to $\pm 15 \%$ of the range of values (e.g. $a_{i j} \leftarrow a_{i j} \mathrm{U}(-1.5,1.5)$ when 10 items are available $)$.

For each of these settings we instantiated 40 matrices: 20 matrices with background values following a Uniform distribution, $\mathrm{U}(1,|\mathcal{L}|)$, and 20 matrices with background values generated according to a Gaussian distribution, $\mathrm{N}\left(\frac{|\mathcal{L}|}{2}, \frac{|\mathcal{L}|}{6}\right)$. The performance of BicPAM is an average across these 40 matrices.

\section{Comparison of biclustering approaches in synthetic data}

We selected five state-of-the-art approaches able to discover biclusters under additive-multiplicative assumptions: FABIA with sparse prior option [2], Bexpa [66], ISA [67], Plaid [6] and OPSM [19]. Additionally, we considered CC [62], Samba [9], xMotifs [18], and three pattern-based biclustering approaches: BiModule [13], DeBi [10] and RAP [14]. Although the last six biclustering approaches use more simplistic homogeneity criteria, their inclusion is critical to study the biological significance of BicPAM's solutions and to test BicPAM's performance improvements when considering biclusters with constant models.

We used the following software to run these methods: R packages fabia [68] and biclust [69], BicAT [70], (Evo-)Bexpa [66] and Expander [71]. The specified number of biclusters for FABIA (with and without sparse equation), Bexpa, CC and ISA (number of starting points) was the number of hidden biclusters plus $10 \%:|\mathcal{H}| \times 1.1$.
Note that this required specification can be used to guide the search space exploration against other biclustering approaches and optimistically bias FABIA consensus (FC) levels. The default number of iterations for OPSM was varied from 10 to 200 iterations. The remaining methods were executed with default parameterizations. For this comparison, BicPAM was parameterized with closed patterns discovered using discretization methods with three distinct sets of items $(|\Sigma| \in\{3,5,7\})$, under a simple merging option (70\% overlap) and filtering of biclusters based on an overlapping area over $30 \%$ against a larger bicluster. Additionally, two items were assigned to values near item-boundaries, leading to an increase in the size of transactions of $8-11 \%$. The support threshold was incrementally decreased $10 \%$ and stopped when the discovered biclusters covered a minimum area of the input matrix $(>5 \% \times|X| \times|Y|)$.

The ability of these approaches to retrieve the hidden biclusters using FABIA data settings is synthesized in Figure $10 . \operatorname{FC}(\mathcal{B}, \mathcal{H})$ was measured across the 100 matrices generated for each setting. BicPAM is the best performer for biclusters following additive models with different signal properties (Wilcoxon-test at $0.01 \%$ ) and, together with FABIA, the best option for multiplicative models. The exhaustive nature of BicPAM searches and the ability to rely on multiple discretization levels without risk of introducing noise (by assignment multiple items for values near ranges-boundaries) support these observations.

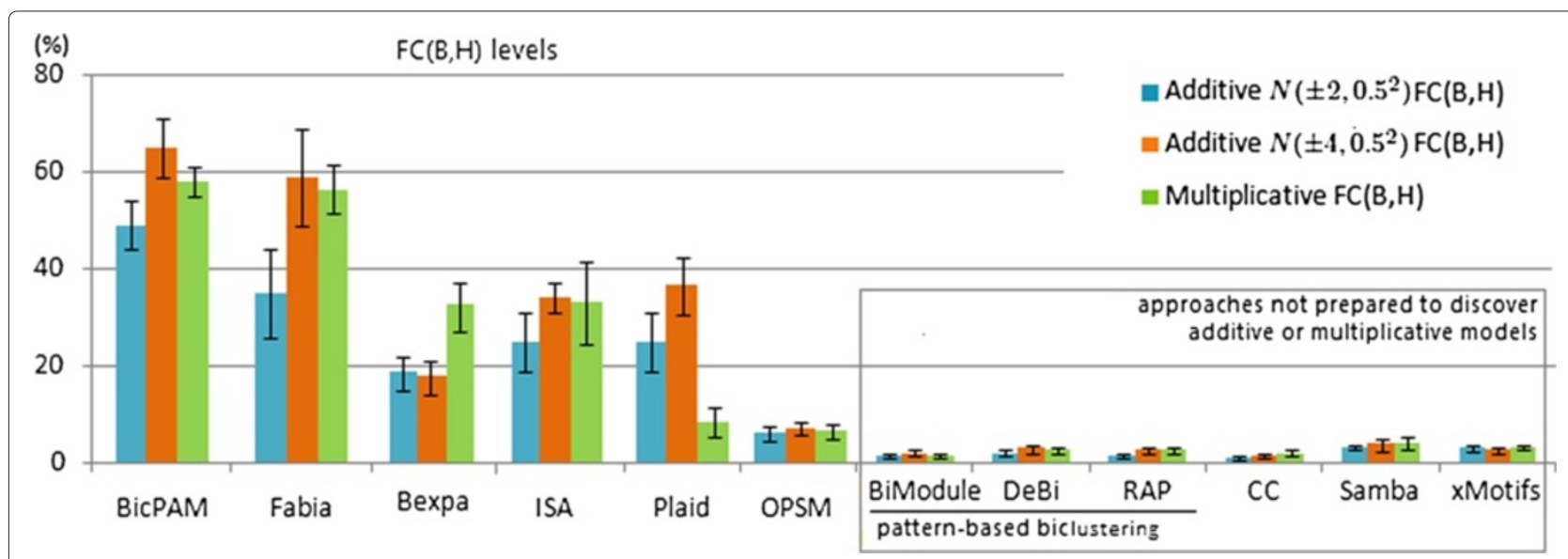

Figure $10 \mathrm{FC}$ levels across biclustering approaches using FABIA datasets. 


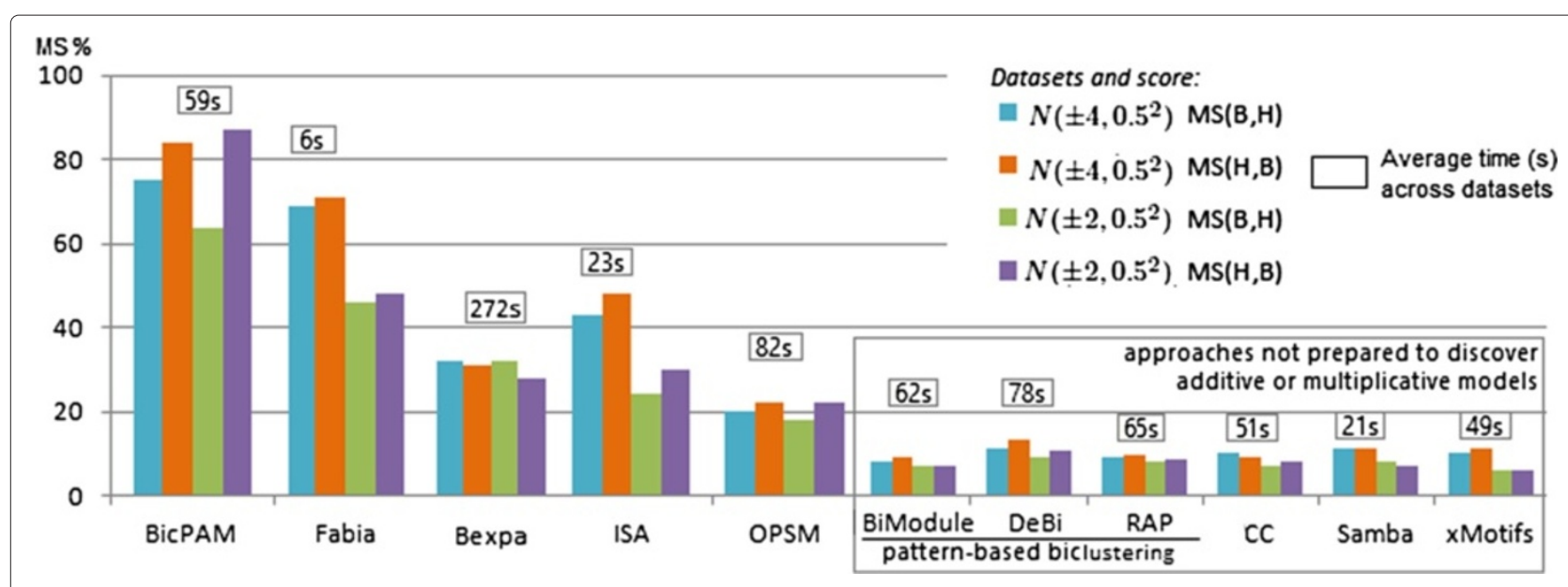

Figure 11 Match scores across biclustering approaches using FABIA datasets.

FABIA is a competitive non-exhaustive alternative, sensitive to the planted noise. Nevertheless, it requires prior knowledge regarding the number of biclusters. Since ISA is tunned to discover biclusters with gradual changes on values, its scoring schema to find modules with selfconsistency is not well suited to discover biclusters modeled by additive signals. Plaid is able to locally identify additive factors. Understandably, the set of approaches not able to discover biclusters with scaling and shifting factors is considerably less effective. The FC levels of OPSM are strongly penalized since OPSM outputs a large number of biclusters with varying sizes (including biclusters with either small number of genes or conditions).

A comparison of match score levels across biclustering approaches when using the FABIA generated datasets is provided in Figure 11. Results confirm the superior performance of BicPAM both in terms of the $\operatorname{MS}(\mathcal{B}, \mathcal{H})$ score (correctness) and $\mathrm{MS}(\mathcal{H}, \mathcal{B})$ score (completeness). BicPAM is able to exhaustively mine the solution space and combine multi-level discretization thresholds. The average efficiency levels of BicPAM show its ability to perform exhaustive searches in useful time for compu- tationally complex settings. FABIA is the most efficient approach.

Figures 12 and 13 assess the ability of the analyzed biclustering approaches to discover planted biclusters with different coherency criteria (using an alphabet with 10 levels of expression) and varying the number of rows and columns (planted according to an Uniform distribution). Figure 12 shows that BicPAM's performance (in the absence of extensions to discover non-constant biclusters) is superior against the three peer pattern-based methods. Figure 13 captures relevant changes in performance when considering additive and multiplicative coherencies. In order to promote the readability of these charts, we excluded the performance of the approaches not prepared to discover biclusters under these assumptions. Results confirm the superior performance of BicPAM in terms of $\operatorname{MS}(\mathcal{B}, \mathcal{H})$, that is, the majority of the discovered biclusters are well described by the hidden biclusters (correctness), and $\operatorname{MS}(\mathcal{H}, \mathcal{B})$, that is, the majority of the hidden biclusters can be mapped into a discovered bicluster (completeness). Although FABIA is the second choice for non-constant coherency, it is not prepared to deal with overlaps and it accommodates high levels of noise since

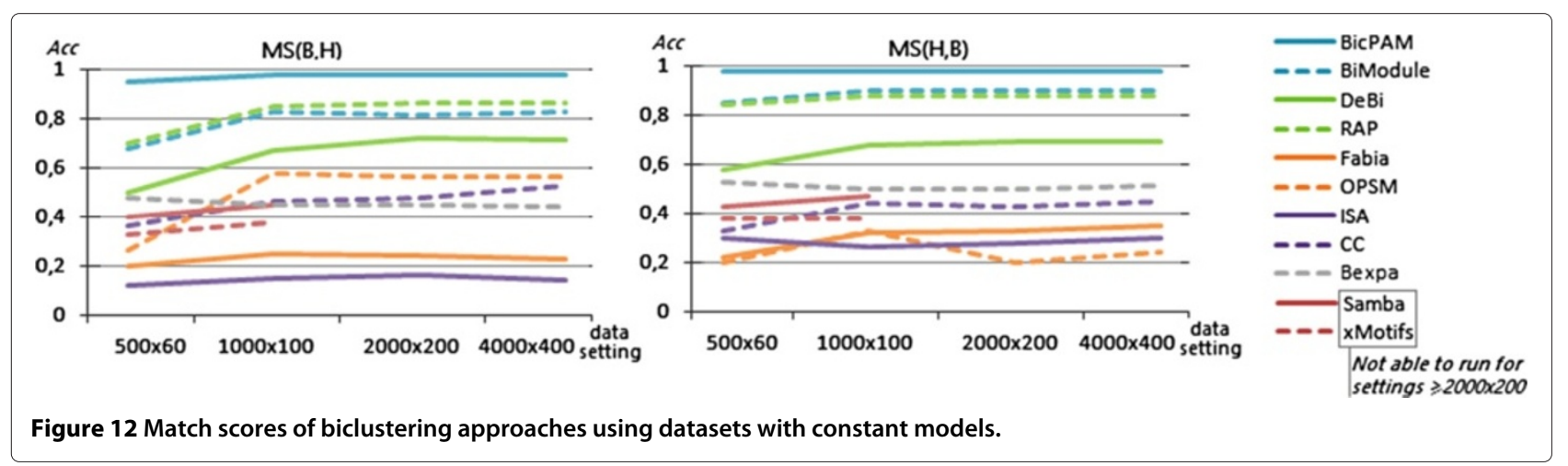




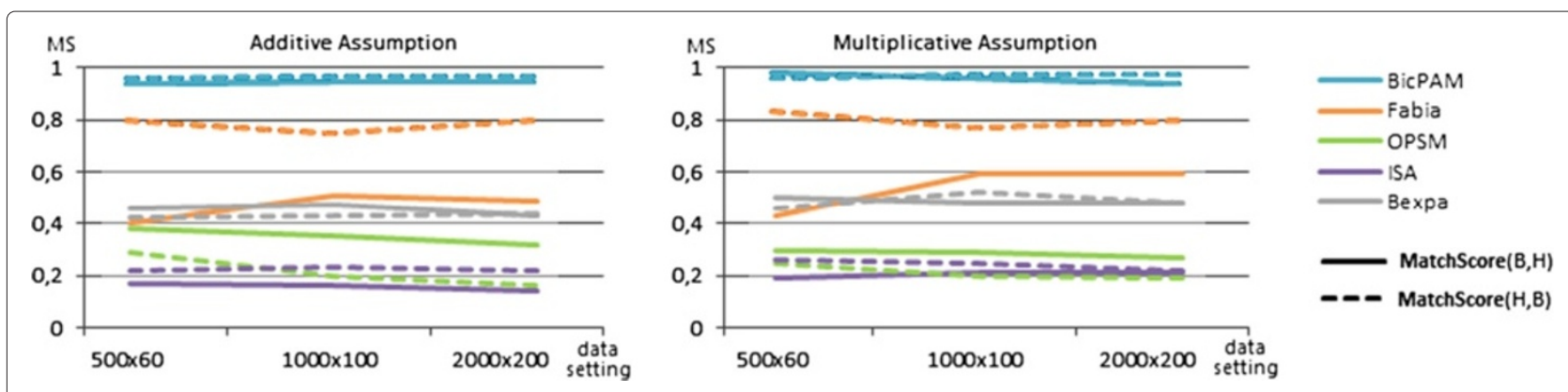

Figure 13 Match scores of biclustering approaches using datasets with non-constant models.

it is not prepared to differentiate all of the 10 levels of expression, resulting in biclusters with a larger number of false positive genes.

Finally, Figure 14 shows that, although all approaches are scalable for medium-sized matrices, efficiency deterioration is faster for OPSM, BicPAM and CC. The efficiency of peer pattern-based approaches is slightly worse than that of BicPAM as they do not seize the benefits of FP-growth searches.

\section{Performance analysis in synthetic data}

In this section we study the efficiency limits of BicPAM. Then we assess the ability of BicPAM to discover different types of biclusters for data with varying regularities. Finally, we go further on understanding the impact of using different strategies related with the mining, mapping and closing steps.

\section{Efficiency limits}

To show the boundaries on BicPAM efficiency we considered matrices with 10.000 rows (magnitude of the human genome). The results are provided in Figure 15. We varied the number of conditions, the number of items $(|\mathcal{L}| \in$ $\{5,7\})$ and the underlying coherency assumptions for this assessment. We consider the default merging procedure for the closing step. We planted 15 biclusters to occupy $2 \%$ of the area of the generated matrices and used Charm algorithm [45], an efficient pattern miner to deliver closed patterns (maximal biclusters). Generally, we observe that BicPAM is able to discover constant biclusters for matrices up to $10.000 \times 350$ and additive/multiplicative biclusters for matrices up to $10.000 \times 200$. Understandably the number of items has strong impact in efficiency as it defines the density of the correspondent itemset database and, therefore, the complexity of the mining step. Note, additionally, that the extensively studied scalability principles based on extensions over pattern mining methods - parallelization, distribution, streaming and error-bounding principles [12] - can be easily included in the mining step of BicPAM to guarantee its scalability over harder data settings.

\section{Recovery of (non-)constant biclusters}

Although BicPAM relies on exhaustive searches, its performance highly depends on the ability to deal with noise, discretization errors and coherency assumptions. Figure 16 shows BicPAM's performance with a parameterizable number of items for the datasets generated under a constant assumption. FC levels are attractive, although they are penalized by the exclusion of rows due to the planted noise, allowed overlapping among planted biclusters together with the fact that the number of discovered biclusters is usually higher than the number of planted biclusters.

A smaller number of items turns the matrix denser, decreasing the efficiency bounds of BicPAM. Using a similar experimental setting, Figure 17 illustrates the

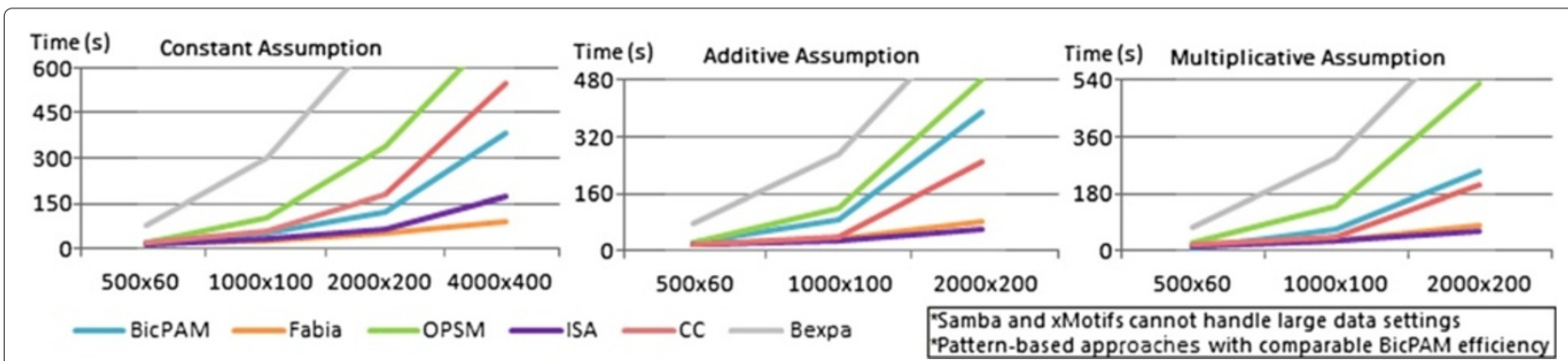

Figure 14 Efficiency of biclustering approaches using the generated datasets. 


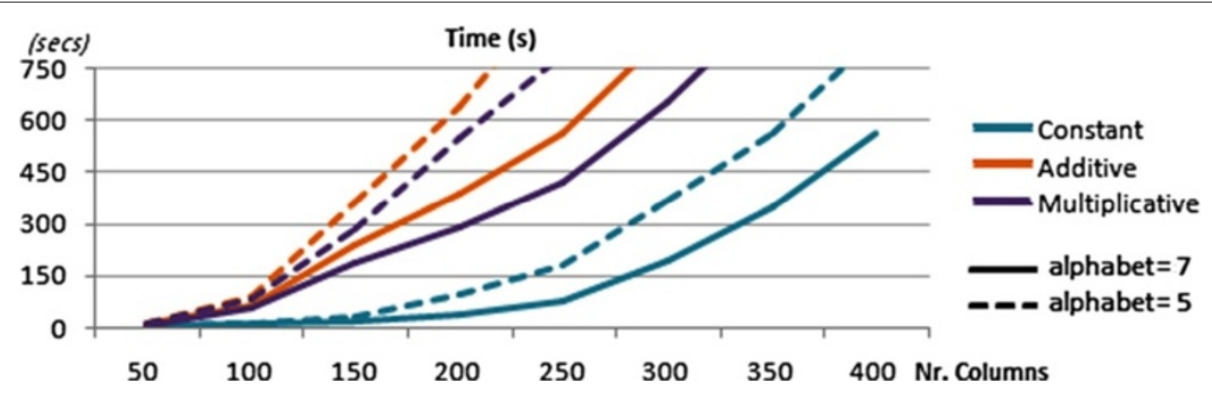

Figure 15 Efficiency bounds of BicPAM for 10000 rows (magnitude of the human genome).

performance of BicPAM for datasets with planted biclusters with an additive assumption. Although the observed FC scores are high, they are worse than for constant datasets due to the higher probability of background values to form a non-planted additive bicluster. Interestingly, although a na?ve search for additive biclusters would cost as much as $|Y|$ times as the search for constant biclusters, the considered pruning fosters efficiency.

Finally, Figure 18 illustrates BicPAM's performance under a multiplicative assumption. Contrasting with the previous analysis, FC levels decrease for the larger matrices as the multiplicative factor is more prone to local mismatchings. This problem can, however, be corrected through closing options. Similarly to the search for additive biclusters, BicPAM seizes efficiency gains by pruning the search space. Additionally, the multiplicative assumption is structurally more efficient than its additive peer since the number of spurious biclusters is considerably low due to the broader range of items observed within each iteration, which leads to sparser matrices.

To complement previous analysis, Figure 19 provides $\operatorname{BicPAM's} \operatorname{MS}(\mathcal{B}, \mathcal{H})$ levels for different levels of expression. The observed MS levels are higher than FC levels due to the absence of penalizations of outputting more biclusters than the number of planted biclusters. In particular, MS levels for medium- to-large datasets are, respectively, above $95 \%, 91 \%$ and $87 \%$ for constant, additive and multiplicative.

A detailed look of BicPAM's performance, when considering 7 items and default noise handling, merging and filtering options, is provided in Table 2. The results are organized according to bicluster type, matrix size (and structure of planted biclusters) and underlying distribution of background values. The slightly worse performance when the input values are generated by a Gaussian distribution is not related with the increased probability of background values to form non-planted biclusters (since values are properly discretized), but with the increased difficulty of modeling the planted biclusters with Uniform values. We found $\operatorname{MS}(\mathcal{B}, \mathcal{H})$ to be lower than $\operatorname{MS}(\mathcal{H}, \mathcal{B})$ since the exhaustive nature of BicPAM leads to at least one found bicluster with a direct correspondence to each hidden bicluster.

\section{Mining options}

Figure 20 illustrates the impact of the algorithmic choice in the efficiency of BicPAM. The three main paradigms for frequent itemset mining (Apriori, FPGrowth, and verticalbased Eclat) were tested based on implementations from SPMF [72] software. These methods were extended in order to be able to deliver the transaction set supporting each frequent itemset. For this assessment we used a discretization step with 10 items and constant planted biclusters based on all frequent patterns. The results were collected for the $1000 \times 100$ generated dataset setting. FPGrowth and Eclat are the most competitive choices when dealing with very small support thresholds. In particular, FPGrowth is the best performer for the setting used for supports near and below 1\%. Finally, Apriori is the best option for medium-to-large support levels.

The impact of choosing alternative pattern representations (simple, closed, maximal) in efficiency and MS levels is presented in Figure 21. For this assessment we used three distinct methods: FPGrowth [42] to output simple patterns, Charm [45] to output closed patterns (maximal biclusters) and CharmMFI [45] to output maximal patterns. Similarly, we considered the $1000 \times 100$ setting and 10 items.

Three main observations can be retrieved from this analysis. First, the use of maximal patterns for biclustering should be avoided as it gives preference to biclusters with a large number of columns and discards biclusters with a subset of these columns (even when they have a larger number of rows). Understandably, this penalizes the $M S(\mathcal{H}, \mathcal{B})$ levels. $M S(\mathcal{B}, \mathcal{H})$ scores are not so affected as each maximal bicluster is covered by a planted bicluster. Second, the use of simple patterns for biclustering can degrade the $M S(\mathcal{B}, \mathcal{H})$ in comparison with closed patterns. This score penalizes the discovery of biclusters 

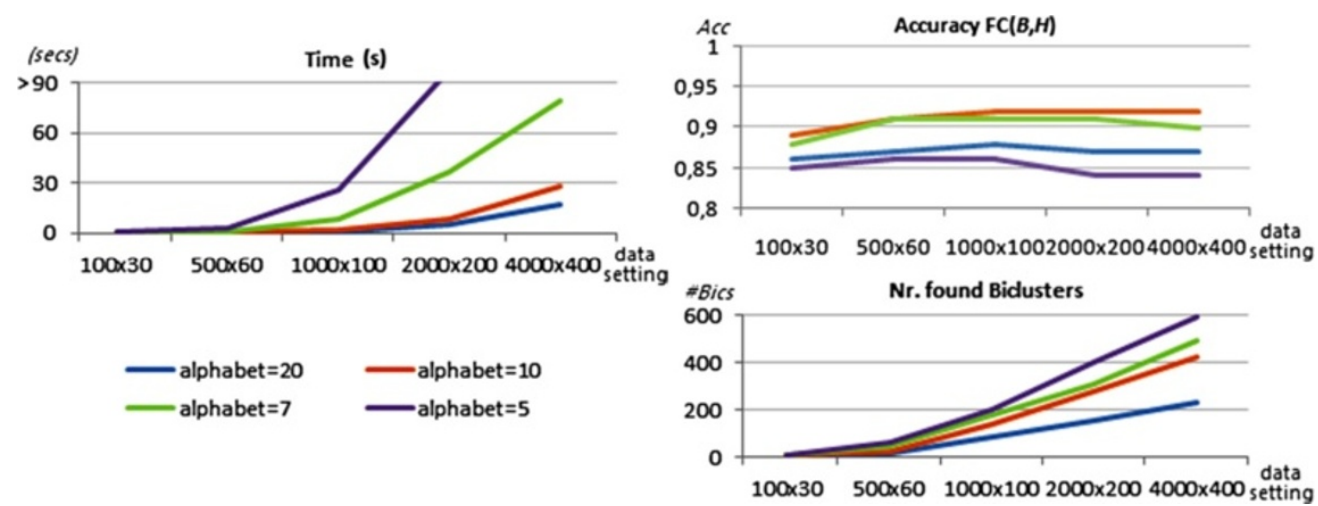

Figure 16 Performance of BicPAM under a constant assumption.
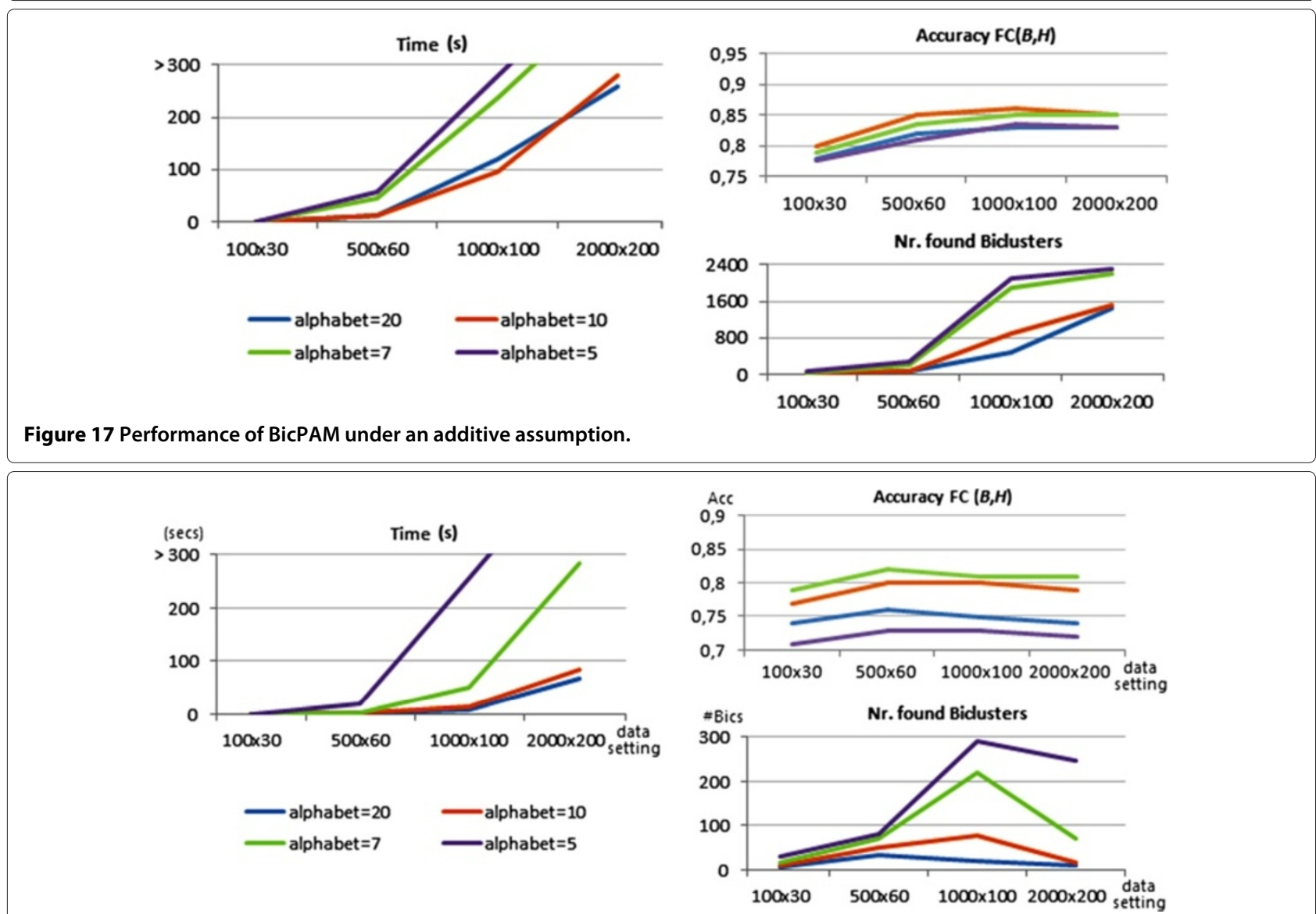

Figure 18 Performance of BicPAM under a multiplicative assumption.

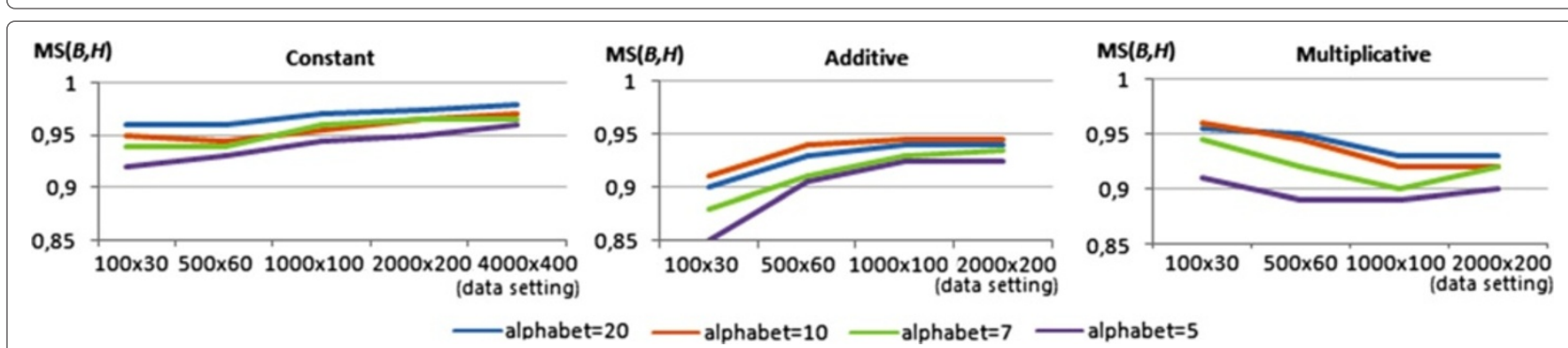

Figure 19 Match score levels of BicPAM under constant, additive and multiplicative assumptions. 
Table 2 FC and MS levels of BicPAM in different settings (mean and variance from 20 datasets)

\begin{tabular}{|c|c|c|c|c|c|c|c|c|c|}
\hline \multirow[b]{2}{*}{ Metric } & \multirow[b]{2}{*}{ Coherency } & \multicolumn{2}{|c|}{$100 \times 30$} & \multicolumn{2}{|c|}{$500 \times 60$} & \multicolumn{2}{|c|}{$1000 \times 100$} & \multicolumn{2}{|c|}{$2000 \times 200$} \\
\hline & & Normal & Uniform & Normal & Uniform & Normal & Uniform & Normal & Uniform \\
\hline \multirow{3}{*}{ FC } & Constant & $0.862 \pm 0.017$ & $0.930 \pm 0.014$ & $=0.018$ & 0.95 & $0.909=$ & 006 & $0.907 \pm 0.014$ & 0.011 \\
\hline & 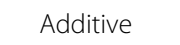 & $0.782 \pm 0.021$ & $0.831 \pm 0.008$ & $0.834 \pm 0.014$ & $0.888 \pm 0.007$ & $0.845 \pm 0.018$ & $0.897 \pm 0.007$ & $0.827 \pm 0.015$ & $0.887 \pm 0.006$ \\
\hline & Multiplic & $0.762 \pm 0.028$ & $0.794 \pm 0.013$ & $0.790 \pm 0.019$ & $0.825 \pm 0.014$ & $0.785 \pm 0.020$ & $0.840 \pm 0.011$ & $0.767 \pm 0.020$ & $0.819 \pm 0.015$ \\
\hline \multirow{3}{*}{$\mathrm{MS}(\boldsymbol{\mathcal { B }}, \mathcal{H})$} & $r$ & 8 & 7 & 2 & 0 & 0 & 05 & 1 & .008 \\
\hline & Additive & $0.895 \pm 0.017$ & $0.945 \pm 0.006$ & $0.925 \pm 0.012$ & $0.963 \pm 0.003$ & $0.913 \pm 0.008$ & $0.981 \pm 0.007$ & $0.917 \pm 0.011$ & $0.974 \pm 0.006$ \\
\hline & Multiplicative & $0.902 \pm 0.019$ & $0.958 \pm 0.014$ & $0.906 \pm 0.015$ & $0.953 \pm 0.009$ & $0.910 \pm 0.015$ & $0.941 \pm 0.008$ & $0.886 \pm 0.019$ & $0.948 \pm 0.010$ \\
\hline \multirow{3}{*}{$M S(\mathcal{H}, \mathcal{B})$} & Constant & $0.956 \pm 0.013$ & $0.984 \pm 0.006$ & $0.960 \pm 0.007$ & $0.981 \pm 0.004$ & $0.961 \pm 0.004$ & $0.996 \pm 0.002$ & $0.957 \pm 0.009$ & $0.993 \pm 0.002$ \\
\hline & Additive & $0.955 \pm 0.012$ & $0.997 \pm 0.001$ & $0.959 \pm 0.006$ & $0.997 \pm 0.002$ & $0.955 \pm 0.004$ & $0.995 \pm 0.002$ & $0.957 \pm 0.007$ & $0.995 \pm 0.00$ \\
\hline & Multiplicative & $0.937 \pm 0.015$ & $0.966 \pm 0.008$ & $0.924 \pm 0.012$ & $0.968 \pm 0.008$ & $0.923 \pm 0.010$ & $0.963 \pm 0.009$ & $0.927 \pm 0.013$ & $0.974 \pm 0.00$ \\
\hline
\end{tabular}

contained in larger planted biclusters, even when the discovered biclusters have a heightened homogeneity. Third, the search for closed and maximal patterns is slightly more efficient than the search for simple patterns as a result of additional pruning procedures. These observations support the use of closed patterns. Furthermore, they correspond to maximal biclusters, which are in general the aim of effective biclustering algorithms $[1,13,73]$.

\section{Mapping options}

In order to assess the impact of the proposed mapping strategies to handle missing values (Figure 6), we randomly removed a varying number of elements from the generated matrices for the $1000 \times 100$ setting. Figure 22 illustrates how the performance of BicPAM (using Charm and 10-item discretization) varies with a percentage of missings ranging from 0 to $10 \%$ (that is, from 0 to 10.000 elements). Note that $10 \%$ is already considered a very critical number of missings that may compromise the ability to retrieve the true biclusters. We observe that this problem can be mitigated recurring to the proposed BicPAM missing handlers.

When analyzing the results in Figure 22, three observations can be retrieved. First, $M S(\mathcal{B}, \mathcal{H})$ under the baseline strategy (remove the missings) significantly decreases from $97 \%$ to near $70 \%$ when the percentage of missings reaches $10 \%$. Although this solution is easily implemented in BicPAM (removing an element from respective transactions), the majority of existing biclustering algorithms only allow for removals on the columns or the rows where a missing occurs (impracticable even in the presence of a few missings as illustrated). Second, the ability to retrieve the planted biclusters increases when considering the nearest 2-3 values against the strategies that consider the closest value only or all the possible values (relaxed strategy). This is justified by two factors: 1) when estimating more than one value for a missing, there is an increased chance to recover the original value and, therefore, of not damaging a planted bicluster; 2) when considering all the possible values for a missing, there is an increased amount of noise that is added and can lead to the emergence of false biclusters. Third, although inserting multiple values to replace a missing is an attractive option in terms of accuracy, its efficiency is penalized as the itemized matrix becomes denser (consistent with the number of discovered biclusters). Still, when considering only the closest 2-3 values, scalability is maintained for levels of noise up to $10 \%$.

\section{Closing options}

We planted additional levels of noise to evaluate the closing options. This was performed by changing the values of specific elements by a randomly distant value (distance $>25 \%$ of the domain range). The percentage of

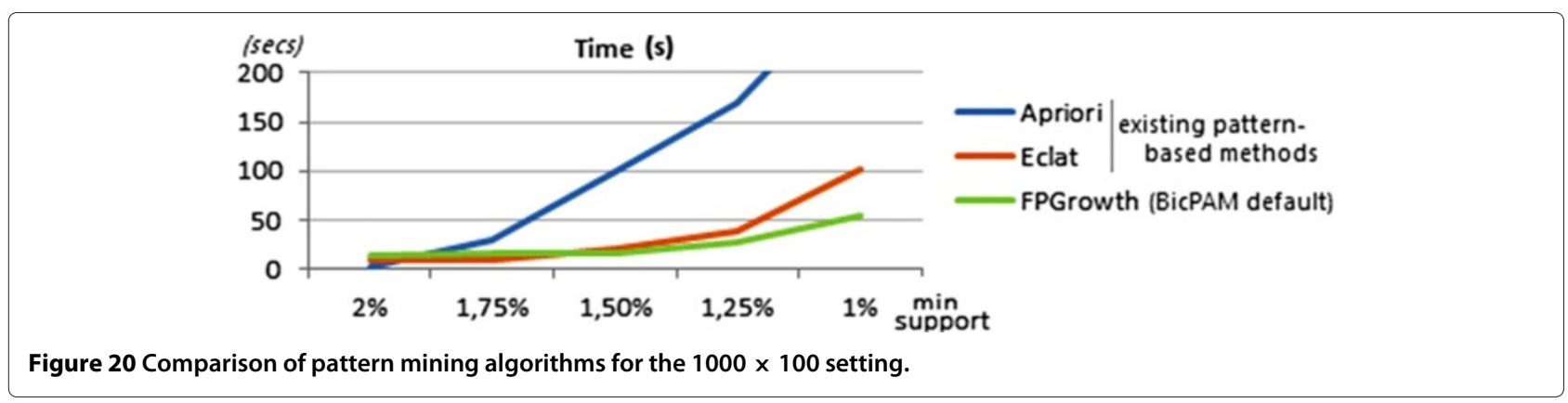




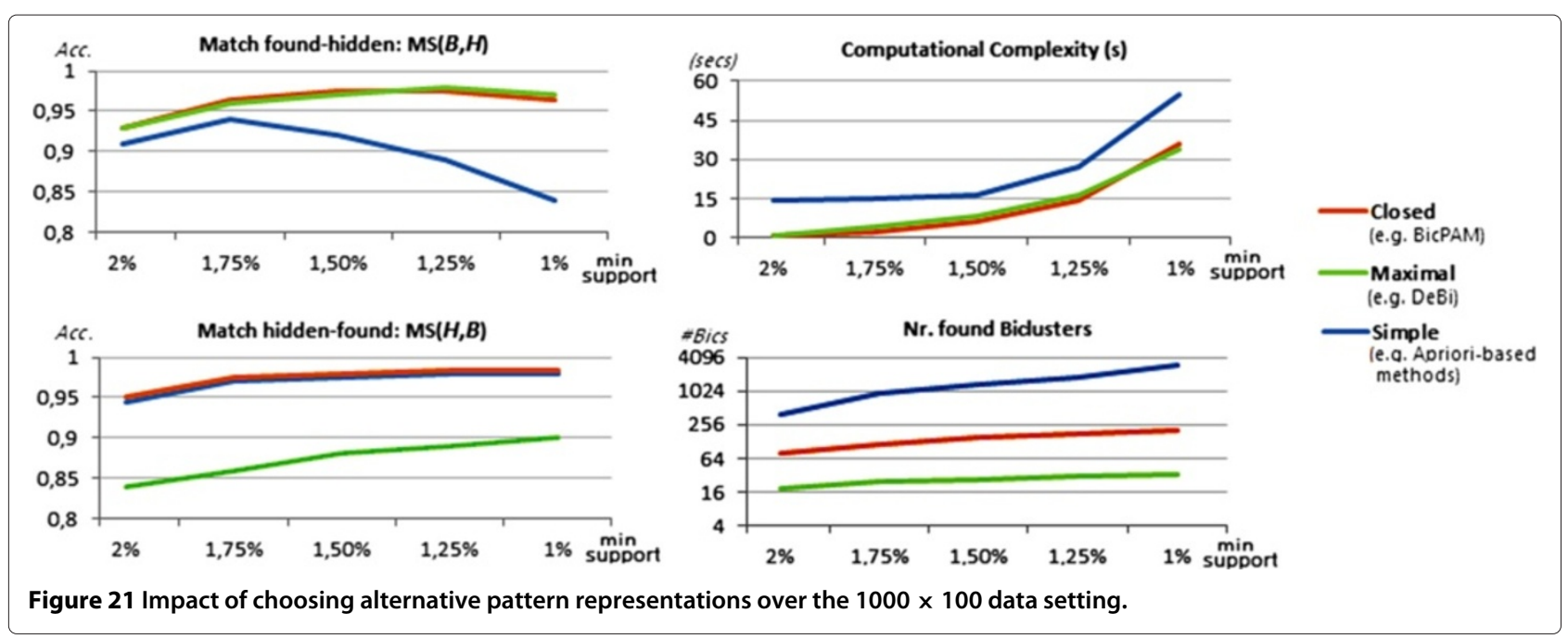

noisy elements was varied from 0 to $10 \%$. We used the $1000 \times 100$ setting, Charm and a total of 10 items.

Figure 23 describes the impact of alternative strategies to extend biclusters. When no noise is planted, mergingbased strategies are able to achieve slightly higher matching scores since they can cover elements originally missed due to discretization errors or by the allowed overlapping among planted biclusters. When increasing the planted noise, the presence of extension options is critical to maintain interesting accuracy levels. Both the inclusion of new rows and columns (recurring to statistical tests or by lowering the support of pattern miners) and the merging of the resulting biclusters are able to maintain match scores above $90 \%$ (20 percentage points higher than the baseline option).

Figure 24(a) illustrates the impact of merging biclusters with large overlapping areas assuming a level of planted noise of $5 \%$. The baseline case corresponds to an overlapping area of $100 \%$. When relaxing the overlapping criteria, $M S(\mathcal{B}, \mathcal{H})$ (and also $M S(\mathcal{H}, \mathcal{B})$ ) increases, as the merging step allows for the recovery of missing rows and columns. However, this improvement in behavior is only observable until a certain overlapping threshold (near 70\% for this experimental setting). Match scoring decreases below this threshold. A correct identification of the optimum threshold can lead to significant gains (near 15 percentage points for this experimental setting).

Finally, the use of filtering strategies can also lead to an enhanced ability to recover the planted biclusters. Although the filtering of biclusters with weak homogeneity impacts accuracy, this analysis targets the removal of rows and columns (on each bicluster) that do not satisfy a specific homogeneity threshold. Figure 24(b) illustrates the impact of removing potentially false rows and columns assuming a level of planted noise of $2 \%$. The impact is only significant when considering a low-to-medium number of items, since for these cases filtering is able to correct the errors related with the large ranges of values per item that

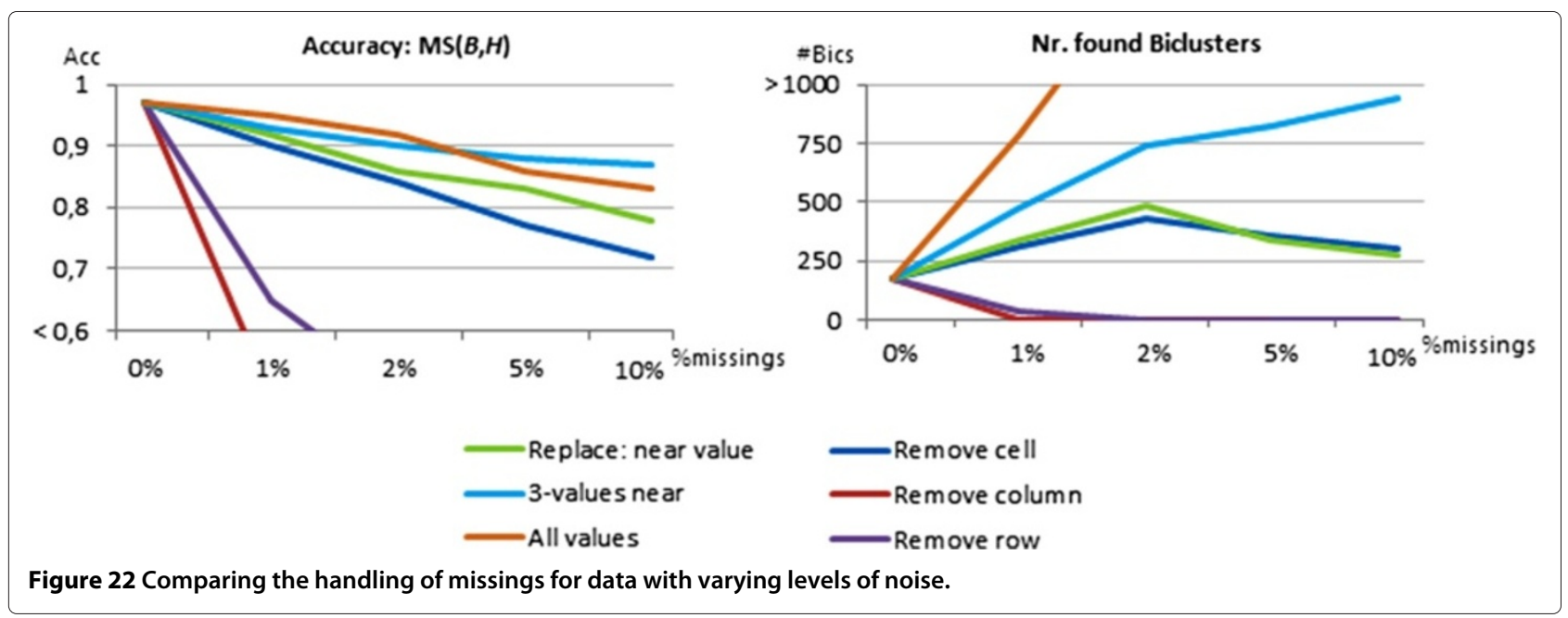




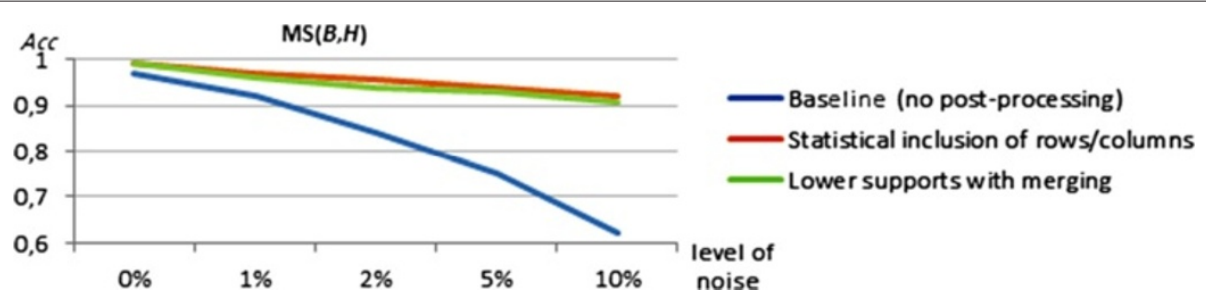

Figure 23 Impact of extending biclusters for data with varying levels of noise.

lead to false biclusters. Similarly to the merging option, an increase in the matching score is observed when compared to the baseline case (an homogeneity degree of $0 \%$ ) up to $75 \%$, given by $1-M S R$ [62]. From this upper threshold the match scores decrease since the homogeneity criteria becomes too restrictive.

\section{Results in real data}

To assess the performance of BicPAM in real data, we compared the biological significance of BicPAM's solutions against state-of-the-art biclustering solutions using three distinct gene expression datasets [74,75]: 1) dlblc dataset (660 genes, 180 conditions) to study responses to chemotherapy [76], 2) hughes dataset (6300 genes, 300 conditions) to characterize nucleosome occupancy [77], and 3) gasch dataset (6152 genes, 176 conditions) to measure Yeast responses to environmental stimuli [78]. For the gasch dataset, we considered the multiple time points per condition and averaged the replicates of the steady state. The missing values were not removed since BicPAM can cope with them. For the state-of-theart biclustering approaches, we maintained the parameterizations used in the previous section. In particular, pattern-based approaches were parameterized with multiple levels of expression $(|\mathcal{L}| \in\{4 . .7\})$. BicPAM output include constant, additive, multiplicative and symmetric biclusters, discovered under different closing options. The selected closing options were: merging (70\% overlap); relaxed merging (55\% overlap) with filtering of rows; and tight merging (90\% overlap) with extensions on rows that appear in another bicluster sharing a minimum 50\% of the conditions. In what follows, we analyze the results obtained focusing the three following points: 1) functional enrichment, 2) transcriptional regulation, and 3) coherence.

\section{Functional enrichment}

The biological relevance of the biclusters from the different biclustering solutions was obtained using the Gene Ontology (GO) annotations computed by GoToolBox [79]. To discover the enriched GO terms, we computed the p-values obtained using the hypergeometric distribution to access the over-representation of a specific term. In order to consider a bicluster to be significant, we require its genes to show enrichment in one or more of the "biological process" ontology terms by having a (Bonferroni corrected) p-value below 0.05 .

Table 3 provides a compact view on the biological significance of the compared approaches. BicPAM is able to discover the largest number of (non-similar) biclusters with significantly enriched terms for each dataset. The analysis of these terms against the significant terms found in other biclustering solutions shows the completeness of BicPAM's solutions (as they cover the majority of the gathered biological functions per dataset), together with the exclusivity and relevance of BicPAM solutions (as they model biclusters with significantly enriched GO-terms that are not discovered by the remaining approaches).
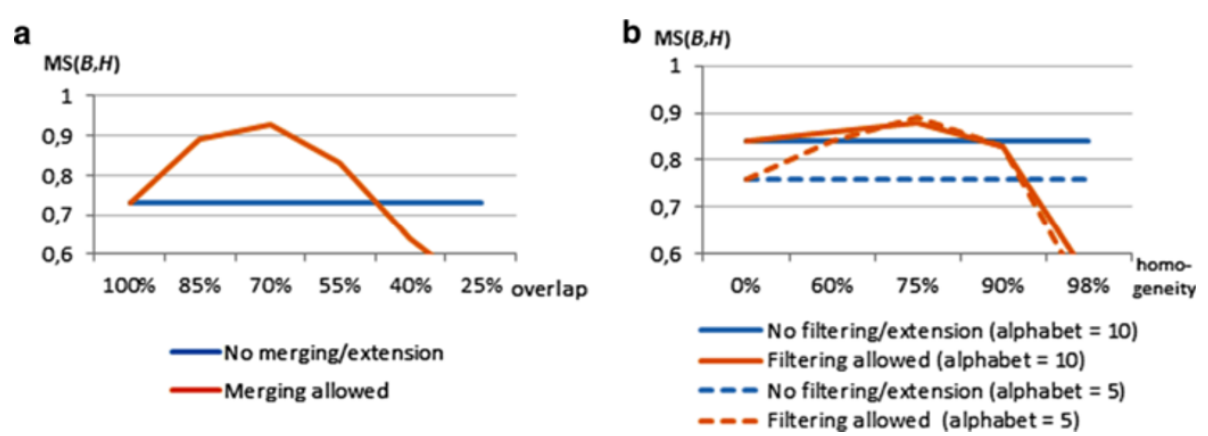

Figure 24 Impact of merging and filtering (reduction) for the 1000 × 100 setting. (a) Merging for varying overlapping degrees (5\% of planted noise). (b) Filtering for varying homogeneity degrees ( $2 \%$ of planted noise). 
Table 3 Comparing the biological relevance and novelty of different biclustering solutions

\begin{tabular}{|c|c|c|c|c|c|}
\hline Dataset & Approach & $\sharp$ Bics & $\begin{array}{l}\text { Avg. } \sharp \text { Genes } \\
x \sharp \text { Conds }\end{array}$ & $\begin{array}{l}\# \text { Bics sig. } \\
\text { enriched }\end{array}$ & Coverage and exclusivity of enriched GO terms \\
\hline \multirow{10}{*}{$\begin{array}{l}\text { dlblc } \\
\text { (human } \\
\text { genome) }\end{array}$} & BicPAM & 56 & $83 \times 7$ & $43(77 \%)$ & Highest number of exclusively enriched terms (partial list in Table 4). \\
\hline & BiModule & 322 & $62 \times 4$ & $79(25 \%)$ & Absence of closing options leads to redundant and less significant terms. \\
\hline & DeBi & 31 & $73 \times 6$ & $21(68 \%)$ & Loss of relevant terms due to the inability to discover all maximal biclusters. \\
\hline & $\mathrm{CC}$ & 10 & $41 \times 33$ & $5(50 \%)$ & Exclusive bicluster related with circulatory \& cardiovascular system development. \\
\hline & ISA & 72 & $23 \times 8$ & $8(11 \%)$ & Exclusive bicluster for extracellular structure organization and heparin binding. \\
\hline & Plaid & 3 & $12 \times 49$ & $1(33 \%)$ & Majority of genes modeled in a single background bicluster with general terms. \\
\hline & Fabia & 10 & $79 \times 35$ & $6(60 \%)$ & Small bicluster with superior enrichment of antigen binding functions. \\
\hline & Bexpa & 10 & $16 \times 87$ & $2(20 \%)$ & Small sets of genes supported by large number of conditions. \\
\hline & Samba & 100 & $17 \times 6$ & $18(18 \%)$ & Dedicated terms for antigen processing, peptide cross-linking and disassembly. \\
\hline & OPSM & 12 & $128 \times 5$ & $5(42 \%)$ & $\begin{array}{l}\text { High variance of } \sharp \text { genes and } \sharp \text { conditions; some of the biclusters with low } \sharp \text { genes } \\
\text { (coherency across high } \sharp \text { conditions) have exclusive significantly enriched terms. }\end{array}$ \\
\hline \multirow{10}{*}{$\begin{array}{l}\text { hughes } \\
\text { (yeast } \\
\text { genome) }\end{array}$} & BicPAM & 47 & $360 \times 7$ & $38(81 \%)$ & Exclusive enriched terms due to flexible coherency and post-processing criteria. \\
\hline & BiModule & 219 & $285 \times 4$ & $43(20 \%)$ & Terms with lower sig. than terms from noise-tolerant BicPAM solutions. \\
\hline & DeBi & 28 & $317 \times 7$ & $21(75 \%)$ & Terms observed across very small sets of conditions $(\leq 5)$ are not enriched. \\
\hline & $\mathrm{CC}$ & 10 & $228 \times 58$ & $6(60 \%)$ & GO terms covered by BicPAM constant biclusters. \\
\hline & ISA & 8 & $120 \times 4$ & $5(63 \%)$ & Small biclusters with exclusive significance GO terms: spindle pole and karyogamy. \\
\hline & Plaid & 8 & $78 \times 39$ & $3(38 \%)$ & One bicluster with higher significance for fungal-type cell wall assembly. \\
\hline & Fabia & 10 & $210 \times 49$ & $5(50 \%)$ & Higher significance observed for actin cortical patch and oxidoreductase GO-terms. \\
\hline & Bexpa & 72 & $42 \times 49$ & $1(10 \%)$ & Low number of enriched terms (probably due to the low $\sharp$ genes per bicluster). \\
\hline & Samba & 120 & $18 \times 9$ & $11(9 \%)$ & Enriched terms covered by pattern-based biclustering solutions. \\
\hline & OPSM & 6 & $531 \times 4$ & $3(50 \%)$ & Exclusive bicluster for the negative regulation of metabolic processes. \\
\hline \multirow{9}{*}{$\begin{array}{l}\text { gasch } \\
\text { (yeast } \\
\text { genome) }\end{array}$} & BicPAM & 149 & $411 \times 8$ & $123(83 \%)$ & Large diversity of highly significant GO-terms (partial list in Table 4). \\
\hline & BiModule & 653 & $287 \times 4$ & $159(24 \%)$ & Large but incomplete set of GO-terms as it excludes non-constant biclusters. \\
\hline & $\mathrm{DeBi}$ & 82 & $310 \times 6$ & $61(74 \%)$ & Significance of terms slightly differ than BicPAM due to the handling of noise. \\
\hline & $\mathrm{CC}$ & 10 & $203 \times 79$ & $7(70 \%)$ & Enriched terms appear in BicPAM solutions with higher significance. \\
\hline & ISA & 23 & $292 \times 22$ & $18(78 \%)$ & Enriched terms covered by pattern-based biclustering solutions. \\
\hline & Plaid & 6 & $48 \times 12$ & $3(50 \%)$ & Biclusters (apart from background layer) with lower enrichments than peers. \\
\hline & Fabia & 10 & $310 \times 41$ & $8(80 \%)$ & Bicluster with higher sig. for specific proteasome complexes. \\
\hline & Bexpa & 10 & $63 \times 29$ & $3(33 \%)$ & The few biclusters with deviation in size (higher $\sharp$ genes) are significant. \\
\hline & OPSM & 16 & $212 \times 8$ & $11(69 \%)$ & One bicluster with higher significance for pre-ribosome functions. \\
\hline
\end{tabular}

Although peer pattern-based solutions also find a large number of biclusters with significantly enriched terms, these terms have lower significance. This is due to the fact that these approaches do not provide noise-correction procedures to minimize the item-boundaries problem and cannot discover non-constant biclusters. Additionally, the remaining biclustering solutions provide incomplete sets of GO-terms since their algorithms are not able to deliver flexible biclustering structures with multiple coherencies. Moreover, some of these approaches are neither able to discover biclusters with multiple levels of expression (or homogeneity levels) nor postprocess the raw biclustering solutions. Still, some of the compared approaches were able to deliver a few small biclusters whose terms are more significant than those found with BicPAM. Subsequent analyzes (Tables 4, 5 and 6) provide further empirical evidence for the relevance, completeness and exclusivity of BicPAM solutions.

Table 4 shows the number of biologically significant biclusters found by BicPAM when using closing strategies. In this analysis, a bicluster is considered to be highly significant if it has at least one enriched term with a corrected p-value below 0.01 . To complement this analysis, Table 5 lists some of the most significant biological processes associated with these enriched terms for each data setting [80].

Table 6 shows an illustrative set of the found patternbased biclusters with statistical relevance. Such biclusters 
Table 4 Summary on the biological relevance of BicPAM's biclusters

\begin{tabular}{|c|c|c|c|c|c|c|}
\hline Dataset & Closing option & $\sharp$ Bics & Avg. Area & $\sharp$ Filtered bics & $\sharp$ Highly sig. bics & $\sharp$ Sig. bics \\
\hline & merging & 4803 & $81 \times 7$ & 28 & 22 & 5 \\
\hline \multirow[t]{3}{*}{$d l b / c$} & relaxed merging + reductions & 980 & $83 \times 9$ & 24 & 19 & 3 \\
\hline & tight merging + extensions & 7652 & $79 \times 6$ & 27 & 25 & 2 \\
\hline & merge & 6311 & $432 \times 6$ & 36 & 19 & 12 \\
\hline \multirow[t]{3}{*}{ hughes } & relaxed merging + reductions & 1259 & $492 \times 7$ & 22 & 12 & 8 \\
\hline & tight merging + extensions & 9210 & $398 \times 5$ & 39 & 22 & 11 \\
\hline & merge & 27031 & $392 \times 8$ & 89 & 66 & 12 \\
\hline \multirow[t]{2}{*}{ gasch } & relaxed merging + reductions & 2177 & $486 \times 11$ & 67 & 49 & 11 \\
\hline & tight merging + extensions & 52123 & $367 \times 7$ & 92 & 79 & 9 \\
\hline
\end{tabular}

Table 5 Terms highly enriched in BicPAM's biclusters

\begin{tabular}{|c|c|c|c|c|}
\hline Dataset & ID & Terms & $\begin{array}{l}\text { Bicluster with } \\
\text { best } p \text {-value }\end{array}$ & $\sharp$ Genes \\
\hline \multirow{8}{*}{$d l b / c$} & Dl1 & translational elongation; cytosolic part; translational initiation & $4.49 \mathrm{E}-5$ & 81 \\
\hline & $\mathrm{D} 12$ & Golgi apparatus; MHC protein complex & $5.40 \mathrm{E}-5$ & 83 \\
\hline & $\mathrm{D} 13$ & defense response; receptor activity; single organism signaling; vacuole; cell communication & $4.91-5$ & 162 \\
\hline & $\mathrm{D} 14$ & immune response; response to interferon-gamma & $1.06 \mathrm{E}-4$ & 58 \\
\hline & $\mathrm{D} 15$ & immune system process & 1.27E-4 & 52 \\
\hline & Dl6 & response to interferon-gamma; cellular response to chemical stimulus; response to cytokine stimulus & 0.001 & 60 \\
\hline & $\mathrm{Dl} 7$ & membrane-enclosed lumen; cell division; cell cycle process & $2.92 \mathrm{E}-12$ & 81 \\
\hline & $\mathrm{D} 18$ & small molecule binding; catalytic activity; cell cycle process & $6.14 \mathrm{E}-8$ & 108 \\
\hline \multirow{6}{*}{ hughes } & $\mathrm{H} 1$ & mitochondrion organization; organellar ribosome; mitochondrial matrix; mitochondrial translation & $2.70 \mathrm{E}-39$ & 416 \\
\hline & $\mathrm{H} 2$ & cell periphery; cell wall constituent; oxidoreductase activity; cell wall organization; sexual sporulation & $1.73 \mathrm{E}-4$ & 370 \\
\hline & $\mathrm{H} 3$ & ribonucleoprotein complex biogenesis; nucleus & $3.61 \mathrm{E}-30$ & 426 \\
\hline & $\mathrm{H} 4$ & cellular amino acid metabolic/biosynthetic process; carboxylic acid metabolic/biosynthetic process & $1.3 \mathrm{E}-25$ & 581 \\
\hline & $\mathrm{H} 5$ & organonitrogen compound metabolic process; sulfur compound metabolic process & $1.62 \mathrm{E}-4$ & 504 \\
\hline & $\mathrm{H} 6$ & macromolecular complex; intracell. non-membrane-bounded organelle; membrane-enclosed lumen & 4.80E-14 & 512 \\
\hline \multirow{17}{*}{ gasch } & G1 & nitrogen compound metabolic proc;; carboxylic/organic amino acid processes; structural cytoskeleton & $1.84 \mathrm{E}-16$ & 434 \\
\hline & G2 & cellular carbohydrate metabolic process; cytoplasm & 2.01E-7 & 265 \\
\hline & G3 & generation of precursor metabolites and energy; tricarboxylic acid cycle & $1.16 \mathrm{E}-14$ & 954 \\
\hline & G4 & endomembrane system; retrotransposon nucleocapsid; pore; viral procapsid maturation & $4.34 \mathrm{E}-6$ & 102 \\
\hline & G5 & nucleolus; ncRNA metabolic process & $1.03 \mathrm{E}-61$ & 611 \\
\hline & G6 & intracell. non-membrane-bounded organelle; structural molecule activity & 5.33E-76 & 293 \\
\hline & G7 & cytosolic part; ribosomal subunit & $1.61 \mathrm{E}-88$ & 460 \\
\hline & G8 & membrane-enclosed lumen; nuclear lumen; intracell. organelle lumen & 1.17E-47 & 263 \\
\hline & G9 & mitochondrion organization; mitochondrial part; cytoplasmic part; protein complex biogenesis & $2.06 \mathrm{E}-26$ & 592 \\
\hline & G10 & cellular response to oxidative stress; generation of precursor metabolites and energy & $2.37 \mathrm{E}-4$ & 296 \\
\hline & G11 & binding; nuclear part; preribosome & $2.87 \mathrm{E}-11$ & 508 \\
\hline & G12 & cellular process involved in reproduction & 0.001 & 435 \\
\hline & G13 & macromolecular complex; cell part; structural molecule activity & $6.05 \mathrm{E}-29$ & 1442 \\
\hline & G14 & vacuolar transport; chromosome & $5.09 \mathrm{E}-7$ & 606 \\
\hline & G15 & regulation of cellular (macromolecule) biosynthetic process; protein modification process & $2.28 \mathrm{E}-13$ & 1019 \\
\hline & G16 & organic substance catabolic process; carbohydrate metabolic process; cytoplasm & $1.02 \mathrm{E}-15$ & 648 \\
\hline & G17 & ribonucleoprotein complex biogenesis (general) & $1.08 \mathrm{E}-94$ & 784 \\
\hline
\end{tabular}


Table 6 Illustrative set of biclusters with different properties and heightened biological relevance ( $p$-values after Bonferroni correction)

\begin{tabular}{|c|c|c|c|c|c|c|c|}
\hline \multicolumn{2}{|l|}{ Dataset } & \multicolumn{2}{|l|}{ ID } & Pattern & Items & \multicolumn{2}{|l|}{ Closing options } \\
\hline & & B1 & & FAABFFF & $A-F$ & Merging with tig & pping \\
\hline \multirow[t]{3}{*}{$d / b / c$} & & B2 & & AAABCA & $A-C$ & Extensions allowe & tight merging) \\
\hline & & B3 & & $\mathrm{AAA} . . / \mathrm{EEE}$ & $A-E$ & Reducing with hi & ogeneity \\
\hline & & B4 & & EEECEE & $A-E$ & Merging allowed & \\
\hline \multirow[t]{2}{*}{ hughes } & & B5 & & CCDCBCBCC & $A-E$ & Merging with rel & rlapping \\
\hline & & B6 & & AAAAA/../G..G & $A-G$ & Merging with tig & pping \\
\hline \multirow{2}{*}{ gasch } & & B7 & & AAAGGGA & $A-G$ & Merging with tig & pping \\
\hline & & B8 & & AAABACCCAA & $A-E$ & Merging allowed & \\
\hline ID & Type & & $\sharp$ Genes & $\sharp$ Conds & $\sharp p-$ values $<0.01$ & $\sharp p-$ values $[0.01,0.05]$ & Best p-value \\
\hline B1 & constant & & 83 & 7 & 41 & 21 & $1.97 \mathrm{E}-10$ \\
\hline B2 & constant & & 153 & 8 & 9 & 1 & $2.27 \mathrm{E}-12$ \\
\hline B3 & multiplicative & & 119 & 5 & 5 & 18 & $4.12 \mathrm{E}-8$ \\
\hline B4 & constant & & 581 & 6 & 12 & 7 & $1.31 \mathrm{E}-25$ \\
\hline B5 & constant & & 654 & 10 & 16 & 4 & $1.31 \mathrm{E}-17$ \\
\hline B6 & additive & & 476 & 6 & 12 & 10 & $1.92 \mathrm{E}-6$ \\
\hline B7 & multiplicative & & 483 & 7 & 57 & 10 & $1.24 \mathrm{E}-81$ \\
\hline B8 & additive & & 521 & 10 & 17 & 5 & $4.57 \mathrm{E}-12$ \\
\hline
\end{tabular}

could hardly be discovered by peer biclustering methods, since many of them include conditions with multiple degrees of expression (B1, B2 and B5) and non-constant profiles (B8). All of these biclusters have heightened biological significance as observed by the number of highly enriched terms after Bonferroni correction. Interestingly, we also observe that different closing options lead to biclusters with different shapes, even when the number of items is the same (B4 and B5).

Although a detailed biological analysis is out of the scope of this paper, we provide a brief analysis for one bicluster per dataset. The bicluster identified in Tables 6 and 7 as $B 1$, with 83 human genes with coherent expression across 7 samples, was discovered in dlblc using 6 levels of expression (under a Gaussian discretization). These genes showed very low expression (A) on 2 samples, low expression (B) on 1 sample and very high expression (F) on 4 samples. Over $40 \mathrm{GO}$ terms were highly significant, with the top set of terms being related with immune defense responses (e.g. immune system process, regulation of immune system process) and signaling functions associated to immunomodulating agents, such as cytokine. Significant terms related with Golgi and with the formation of membrane-bound compartments imply their critical roles during the induction of innate immune responses after chemotherapy [81]. Similar biclusters are not discovered when the number of expression levels is decreased or when noise relaxations are not included, thus motivating the need for BicPAM. The illustrative biclusters, found in hughes and gasch datasets, concern genes from Saccharomyces cerevisiae analyzed in the context of studying nucleosome occupancy and responses to different stress conditions, respectively. The enriched terms of bicluster B4 are associated with the formation of carboxylic acid and organonitrogen compounds, with optimum enrichment levels found in the presence of moderate noise-tolerance. Bicluster B7 captures genes with coherent expression across multiple time points from three

Table 7 Enriched GO terms of three illustrative BicPAM biclusters

\begin{tabular}{|c|c|c|}
\hline ID & Dataset & Top 4 GO Terms ( $p$-value) \\
\hline \multirow{2}{*}{ B1 } & \multirow{2}{*}{$d \mid b / c$} & Immune response (2.32E-10); immune system process, defense response $(<1 \mathrm{E}-6)$; \\
\hline & & cytokine-mediated signaling pathway (1.33E-7); Golgi apparatus (1.19E-7). \\
\hline \multirow{2}{*}{ B4 } & \multirow{2}{*}{ hughes } & Carboxylic acid biosynthetic process (1.3E-25) and metabolic process (6.12E-16); \\
\hline & & organonitrogen compound biosynthetic process (2.23E-18) and metabolic process (2.71E-13). \\
\hline \multirow{2}{*}{ B7 } & \multirow{2}{*}{ gasch } & Ribonucleoprotein biogenesis and assembly (1.24E-81); cytosolic part (1.22E-57); \\
\hline & & intracell. non-membrane-bounded organelle (1.31E-65); ncRNA metabolic process (1.82E-52). \\
\hline
\end{tabular}


different heat shocks (shocks from 17, 21 and $25^{\circ} \mathrm{C}$ ). The analysis of GO terms shows functions related with the ribonucleoprotein complex ( $p$-value 1.24E-81), associated with the reassembly and protection of small particles during heat stress responses [82]. Interestingly, other biclusters found in gasch are able to capture coherent levels of expression across different stimuli. An example is bicluster B8 that integrates conditions related with nitrogen depletion, heat stress and diauxic shift. B8 has 521 genes, coherent additive levels of expression across 10 conditions, and over 10 highly significant enriched terms.

\section{Transcriptional regulation}

To complement the results on functional enrichment, we analyzed the highly enriched transcription factors (TFs) using the TFCONES database [83] (human genome) and Yeastract database [84] (yeast genome) using a corrected hyper-geometric statistical test.

Consider the illustrative biclusters provided in Table 7. Some of the enriched transcription factors regulating the genes in bicluster $B 1$ (associated with immune system responses in the human genome) include: HCLS1 gene that plays a key role in regulating clonal expansion and deletion in lymphoid cells [85], IRF1 protein that acts as a tumor suppressor and plays a role not only in antagonism of tumor cell growth but also in stimulating an immune response against tumor cells [85], and TRIM22 antiviral protein involved in cell innate immunity [83]. Other highly enriched TFs that regulate proliferation and transformation (tumor supressors) are ANP32A and RUNX3 [85]. The TFs regulating the genes in bicluster $B 4$ have $p$-values below $1 \mathrm{E}-15$ after correction, each regulating from $50 \%$ to $95 \%$ of the genes in bicluster. They are associated with regulatory functions consistent with the enriched terms. Some of these TFs include histidine biosyntehsis (Bas1p), amino acid biosynthesis (Gcn4p), cyclic AMP receptor protein regulation (Sok2p) and other TFs related with the regulation of carboxylic acid and organonitrogen compounds [86]. Consider now bicluster $B 7$ from gasch. Some of the enriched TFs include Sfp1p, Mga2p, Ace2p, Tup1p, Spt10p and Swi5p ( $p$-values below $1 \mathrm{E}-15)$, each regulating $55 \%-97 \%$ of B7's genes. These factors are known to be involved in stress responses as they regulate cooling and oxygen levels (Mga2p), repair cellular damage (Sfp1p and Spt10p), remodel chromatin (Tup1p) and regulate cell wall protection (Swi5p and Ace2p) [86-88]. Finally, consider bicluster $B 8$, whose genes coherently regulate heat, nitrogen depletion and diauxic shifts. Sfp1p, Bas1p, Ste12p and Tec1p were the most significant TFs in this bicluster ( $p$-values $<1 \mathrm{E}-7)$. Sfp1p controls expression of ribosome biogenesis genes in response to stress and DNA-damage response [86]. Bas1p regulates gene expression for biosynthesis pathways such as pathways related with histidine metabolism, which responds to environmental stimuli (e.g. nitrogen) affecting $\mathrm{pH}$ calibration [86]. Finally, Ste12p and Tec1p act together to regulate genes related with invasive growth, whose production is expected under such stress conditions [86].

An extended analysis of the TFs associated with BicPAM's biclusters for the human and yeast genome is provided in Table 8. In this analysis we retrieved the TFs that are more representative - high coverage of the genes in the biclusters - and significant - high functional enrichment $(p$-value $<1 \mathrm{E}-3)$ - for each one of the twenty five distinct biclusters disclosed in Table 5 associated with the dlblc and gasch dataset. In line with the goal of the these experiments [76,78], we observe that the identified TFs are either directly or indirectly related with the responses to chemotherapy (human) $[83,85]$ and stress conditions (yeast) $[84,86]$. This analysis thus further supports the domain-relevance and adequacy of BicPAM.

Consider the enriched TFs provided in Table 8 for a sample set with 8 distinct biclusters found by BicPAM in the dlblc dataset. Different groups of TFs were identified, each associated with a specific chemotherapy outcome. Some of the TFs acting as putative tumor suppressors include: ANP32A, LZTS1 (protein-coding silenced in rapidly metastasizing and metastatic tumor cells), RUNX3 (protein that binds to the core site of leukemia virus, also frequently silenced in cancer), HCLS1 (antigen receptor signaling deletion in lymphoid cells), IRF1 (protein that stimulates immune responses and regulates tumor cell differentiation), HIf1A (gene responsible for tumor angiogenesis and pathophysiology of ischemic disease), HDAC1 (complex interacting with retinoblastoma tumor-suppressor proteins), TCF3 (protein regulating lymphopoiesis as its deletion is associated with lymphoblastic and acute leukima malignancies) $[83,85]$. Other TFs dedicated to regulate cell proliferation include the STAT families, CREG1, MEF2B, ARID5B, and BCL3 [85]. Understandably, we also observed the B-cell lymphoma protein (BCL6 and its paralog coding gene BCL6B) and other leukemia-related disease genes involved in lymphoma pathogenes, such as BCL11A [83]. Complementarily, immune responses are associated with TRIM22 antiviral proteins, CEBPB, NFATC2 complex, and GTF2I for activating immunoglobulin heavy-chain transcription upon B-lymphocyte activation [85].

Finally, consider the enriched TFs provided in Table 8 for a sample set with 17 distinct biclusters found by BicPAM in the gasch dataset. Since a large number of enriched TFs was identified, Table 8 only provides an illustrative set containing TFs regulating over $50 \%$ of the genes associated with each bicluster. Although the enriched TFs regulate very distinct processes (see Table 5), most TFs are activated in stress conditions, namely: Yap1p, Cin $5 p$ and Hap2p during oxidative stress [86]; Gcn4p, Msn2p and Msn4p during amino acid starvation [86]; Hsf1p during 


\begin{tabular}{|c|c|c|}
\hline Dataset & Bic.ID (Table 5) & Highly enriched TFs \\
\hline & Dl1 & BCL11A, LZTS1, GTF21, HCLS1, HDAC1, MBD4, MEF2B, NCOA3, STAT6 \\
\hline & $\mathrm{D} 12$ & ANP32A, HCLS1, IRF1, MNDA, NCOA1, RUNX3, STAT1, TRIM22, TRIP10 \\
\hline & Dl3 & BCL3, TRIM22, ANP32A, ARID5B, CEBPB, CREG1, IRF1, PFDN5, STAT1 \\
\hline \multirow[t]{13}{*}{ dlblc } & $\mathrm{D} \mid 4$ & ANP32A, IRF1, NCOA1, STAT1, TRIM22 \\
\hline & Dl5 & CREG1, IRF1, TRIM22, ANP32A, STAT1 \\
\hline & Dl6 & ANP32A, IRF1, NCOA1, STAT1, TRIM22 \\
\hline & Dl7 & BCL6, BCL6B, HIf1A, ILF2, POU2AF1, SERTAD1, TCF3 \\
\hline & Dl8 & DR1, DRAP1, HIf1A, ILF2, NCOA3, SERTAD1, TMF1, ZNFN1A1 \\
\hline & G1 & Gcn4p, Sfp1p, Ace2p, Tec1p, Ste12p, Ash1p \\
\hline & G2 & Sfp1p, Msn2p, Bas1p, Tec1p, Sok2p, Abf1p, Ash1p, Cst6p \\
\hline & G3 & Sfp1p, Tec1p, Ste12p, Msn2p, Bas1p, Sok2p, Msn4p, Gcn4p \\
\hline & G4 & Snf6p, Tec1p, Ste12p, Rap1p, Sin4p, Abf1p, Snf2p, Ash1p \\
\hline & G5 & Sfp1p, Ace2p, Cst6p, Tup1p, Msn2p, Spt10p, Spt20p \\
\hline & G6 & Hsf1p, Spt23p, Mga2p, Sfp1p, Spt10p, Msn2p, Gcr1p, Gcn4p \\
\hline & G7 & Sfp1p, Swi5p, Tup1p, Spt10p, Spt20p, Gcr1p, Sin3p, Mga2p \\
\hline & G8 & Sfp1p, Swi5p, Cst6p, Tup1p, Spt20p, Ash1p, Spt10p \\
\hline \multirow[t]{9}{*}{ gasch } & G9 & Yap1p, Ace2p, Sfp1p, Msn2p, Ash1p, Msn4p, Abf1p \\
\hline & G10 & Sfp1p, Msn2p, Msn4p, Cst6p, Abf1p, Sok2p, Bas1p \\
\hline & G11 & Snf6p, Tup1p, Snf2p, Cst6p, Sin4p, Rap1p, Swi3p, Hap2p \\
\hline & G12 & Yap1p, Tec1p, Msn2p, Msn4p, Ste12p, Sok2p \\
\hline & G13 & Snf6p, Tup1p, Abf1p, Snf2p, Cst6p, Sin4p \\
\hline & G14 & Sfp1p, Tec1p, Ste12p, Bas1p, Sok2p, Yrm1p \\
\hline & G15 & Ace2p, Sfp1p, Tec1p, Ste12p, Ash1p, Bas1p, Gcn4p, Sok2p \\
\hline & G16 & Cin5p, Gcn4p, Msn4p, Sfp1p, Msn2p, Tec1p, Ste12p, Sok2p \\
\hline & G17 & Sfp1p, Ace2p, Cst6p, Snf6p, Rap1p, Tup1p, Spt10p, Swi5p \\
\hline
\end{tabular}

variable heat shock elements including hyperthermia [86]; Sfp1p during DNA damage [84]; and Spt23p and Mga2p during cooling [87]. The stress conditions are associated with invasive growth (regulated by Tec1p, Ste12p, Ash1p and Sok2p), and with the need for chromatin remodeling (regulated by Snf6p, Snf2p, Spt20p, Tup1p and Swi3p) and DNA repair (regulated for instance by Abf1p and Spt10p) $[84,86]$.

\section{Coherence}

Figure 25 illustrates four biclusters discovered in the gasch dataset, which are related with the response of Yeast genes to heat shock at different time points. BicPAM's behavior is particularly favorable to the discovery of these biclusters, contrasting with other biclustering approaches. In particular, the combination of constant models with symmetries, multiplicative models with symmetries, additive models with several levels of expression, and additive models with symmetries. The analysis of these biclusters shows the relevance of combining multiple levels of expression $(|\mathcal{L}| \geq 5)$ with noise relaxations for the discovery of meaningful biclusters. Additionally, this analysis supports the importance of allowing sign-changes across multiple levels of expression to capture activation and repression mechanisms in regulatory processes.

\section{Comparison of pattern-based biclustering approaches}

In the previous sections, we provided substantial empirical evidence for the improvements of BicPAM performance in comparison with peer pattern-based methods such as BiModule, DeBi and RAP. First, Figures 10 and 11 show the unique ability of BicPAM to discover non-constant biclusters ( $>50$ percentage points in $\mathrm{MS}$ and FC against BiModule, DeBi and RAP). Second, Figure 12 shows improvements in the discovery of constant biclusters related with BicPAM's ability to deal with the items-boundary problem and to adequately postprocess biclustering solutions. Additionally, BicPAM's ability to combine solutions discovered under multiple levels of expression and to discover all the maximal biclusters 


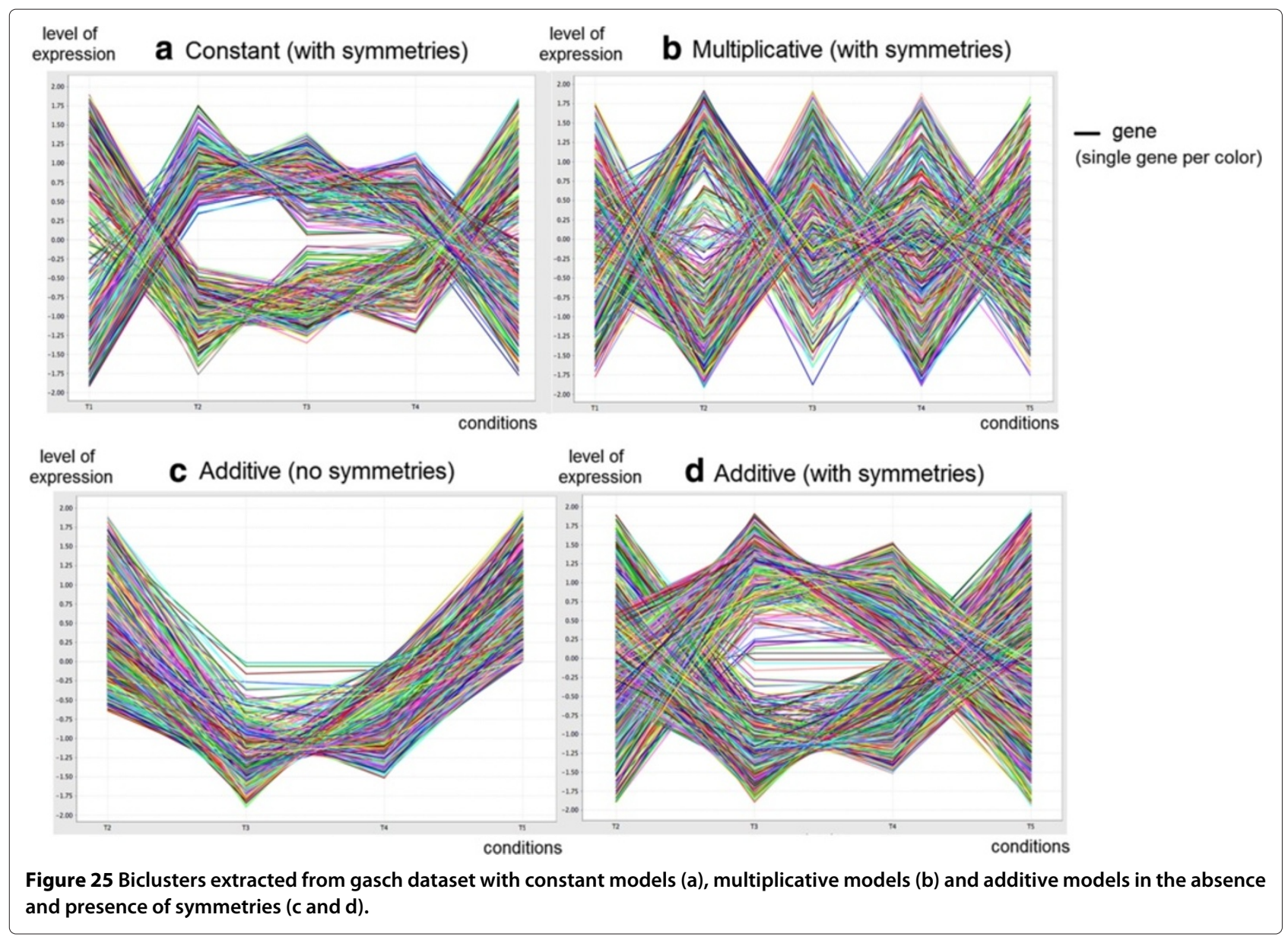

(closed pattern representations) surpasses specific drawbacks found in some of the existing methods. Third, the incorporation of scalability principles and of minimalist FP-trees (Figure 20) guarantee its competitive computational complexity even when procedures to handle noise and adapt the biclustering structures are used. Fourth, Figures 22 to 24 show significant performance improvements of BicPAM due to its exclusive ability to deal with medium-to-high levels of missing values and noise. Finally, the biological relevance of BicPAM's solutions against the solutions provided by the peer methods is assessed in Table 4 and further supported in subsequent analyzes. In particular, we show that BicPAM's solutions cover the (enriched) biological processes associated with peer pattern-based solutions (Table 6). Moreover, they enable the discovery of unique and biologically meaningful biclusters (Tables 5 and 6) such as the four illustrative biclusters in Figure 25.

\section{Conclusion}

A new approach for flexible and robust pattern-based biclustering (BicPAM) is proposed with the goal of performing exhaustive searches to discover biclustering solutions with multiple coherencies under relaxed conditions (arbitrary number and structure of biclusters) with heightened efficiency. BicPAM is the result of integrating existing dispersed contributions on pattern-based biclustering with new critical methods to deal with more flexible expression profiles and to handle varying levels of missing values and noise.

BicPAM goes beyond the constant assumption made by existing pattern-based approaches, and extends the biclustering task to new types of biclusters, including additive and multiplicative assumptions that can accommodate symmetries. It is the first attempt to model these coherencies under a pattern-based approach. This is critical since pattern-based searches are exhaustive, support flexible structures of biclusters, and consider multiple levels of expression (instead of differential expression).

Additionally, BicPAM is able to surpass the common drawbacks related with discretization procedures, since it is able to assign multiple items over a single element to tackle the items-boundary problem. In this way, the transactional database derived from the input matrix can have more items than the number of elements in the original matrix. 
BicPAM relies on dynamic parameterizations for a tuned performance across different settings, including pattern representations, strategies to handle missing values, and postprocessing options for the post-handling of noise and composition of flexible structures. Although the default options are dynamically derived based on the properties of the target dataset, they can also be defined by the user without the need to adapt the core mining task.

Results on both synthetic and real datasets show BicPAM's ability to find optimal solutions over matrices with more than 10.000 rows and up to 400 columns. The assessment of BicPAM's performance against peer patternbased approaches and other state-of-the-art biclustering algorithms supports its heightened flexibility and robustness to noise. Additionally, we observed that the majority of the biclusters discovered by BicPAM in gene expression datasets are functionally relevant and could not be discovered by other biclustering approaches. The analysis of their transcriptional regulation showed significant and meaningful associations.

\section{Software availability}

The datasets and BicPAM executables are available in http://web.ist.utl.pt/rmch/software/bicpam/.

\section{Endnote}

${ }^{a}$ Clustering metrics measure the ability to correctly group rows (or columns), that is, of attaining high intra-cluster similarity and low inter-cluster similarity. Entropy and F-measure metrics are the common choice $[56,57]$. F-measure can be further decomposed in terms of recall (coverage of found rows by a hidden cluster) and precision (absence of rows present in other hidden clusters).

\section{Competing interests}

The authors declare that they have no competing interests.

\section{Authors' contributions}

All the authors were involved in the design of the solution and in the writing of the manuscript. All authors read and approved the final manuscript.

\section{Acknowledgments}

This work was supported by national funds through Fundação para a Ciência e a Tecnologia (FCT), under projects Pest-OE/EEI/ LA0021/2013 and DataStorm (EXCL/EEI-ESS/0257/2012), and the doctoral grant SFRH/BD/75924/2011 to RH.

Received: 18 January 2014 Accepted: 12 November 2014

Published online: 16 December 2014

\section{References}

1. Madeira SC, Oliveira AL: Biclustering algorithms for biological data analysis: a survey. IEEE/ACM Trans. Comput. Biol. Bioinformatics 2004, 1:24-45.

2. Hochreiter S, Bodenhofer U, Heusel M, Mayr A, Mitterecker A, Kasim A, Khamiakova T, Van Sanden S, Lin D, Talloen W, Bijnens L, Göhlmann HWH, Shkedy Z, Clevert DA: FABIA: factor analysis for bicluster acquisition. Bioinformatics 2010, 26(12):1520-1527.
3. Bebek G, Yang J: PathFinder: mining signal transduction pathway segments from protein-protein interaction networks. $B M C$ bioinformatics 2007, 8:335.

4. Ding C, Zhang Y, Li T, Holbrook SR: Biclustering protein complex interactions with a biclique finding algorithm. In ICDM. Washington, DC, USA: IEEE Computer Society; 2006:178-187.

5. Liu J, Wang W: OP-Cluster: clustering by tendency in high dimensional space. In ICDM. Washington, DC, USA: IEEE Computer Society; 2003:187-

6. Lazzeroni L, Owen A: Plaid models for gene expression data. Statistica Sinica 2002, 12:61-86.

7. Odibat $\mathrm{O}$, Reddy $\mathrm{C}$ : A generalized framework for mining arbitrarily positioned overlapping co-clusters. In SDM. Arizona, USA: SIAM 2011:343-354.

8. Zhang L, Chen C, Bu J, Chen Z, Cai D, Han J: Locally discriminative coclustering. Knowl Data Eng IEEE Trans 2012, 24(6):1025-1035.

9. Tanay A, Sharan R, Shamir R: Discovering statistically significant biclusters in gene expression data. Bioinformatics 2002, 18:136-144.

10. Serin A, Vingron M: DeBi: Discovering differentially expressed biclusters using a frequent itemset approach. Algorithms Mol Biol 2011, 6:1-12.

11. Okada $Y$, Okubo K, Horton P, Fujibuchi W: Exhaustive search method of gene expression modules and its application to human tissue data. IAENG IJ Comp Sci 2007, 34:119-126.

12. Han J, Cheng $H$, Xin D, Yan $X$ : Frequent pattern mining: current status and future directions. Data Min. Knowl. Discov. 2007, 15:55-86.

13. Okada Y, Fujibuchi W, Horton P: A biclustering method for gene expression module discovery using closed itemset enumeration algorithm. IPSJ Transactions on Bioinformatics 2007, 48(SIG5):39-48.

14. Pandey G, Atluri G, Steinbach M, Myers CL, Kumar V: An association analysis approach to biclustering. In KDD. New York, NY, USA: ACM; 2009:677-686.

15. Martinez R, Pasquier C, Pasquier N: GenMiner: mining informative association rules from genomic data. In BIBM. Silicon Valley, USA: IEEE CS; 2007:15-22.

16. Yang J, Wang W, Wang H, Yu P: Delta-clusters: capturing subspace correlation in a large data set. In ICDE. San Jose, USA; 2002:517 -528.

17. Califano A, Stolovitzky G, Tu Y: Analysis of gene expression microarrays for phenotype classification. In Proc. Int. Conf. Intell. Syst. Mol. Biol. San Jose, USA; 2000:75-85

18. Murali TM, Kasif S: Extracting conserved gene expression motifs from gene expression data. In Pacific Symposium on Biocomputing. Lihue, Hawaii, USA; 2003:77-88.

19. Ben-Dor A, Chor B, Karp R, Yakhini Z: Discovering local structure in gene expression data: the order-preserving submatrix problem. In RECOMB. New York, NY, USA: ACM; 2002:49-57.

20. Getz G, Levine E, Domany E: Coupled two-way clustering analysis of gene microarray data. Proceedings of the National Academy of Sciences 2000, 97(22):12079-12084.

21. Tang C, Zhang L, Ramanathan M, Zhang A: Interrelated two-way clustering: an unsupervised approach for gene expression data analysis. In BIBE. Washington, DC, USA: IEEE Computer Society; 2001:41-

22. Busygin S, Jacobsen G, KrÃd'mer E, Ag C: Double conjugated clustering applied to leukemia microarray data. In ICDM IW on clustering high dimensional data. Brussels, Belgium: IEEE; 2002.

23. Hartigan JA: Direct clustering of a data matrix. Journal of the American Statistical Association 1972, 67(337):123-129.

24. Sheng Q, Moreau Y, Moor BD: Biclustering microarray data by Gibbs sampling. In ECCB. Paris, France; 2003:196-205.

25. Wang $\mathrm{H}$, Wang $W$, Yang J, Yu PS: Clustering by pattern similarity in large data sets. In SIGMOD. New York, NY, USA: ACM; 2002:394-405.

26. Carmona-Saez P, Chagoyen M, Rodriguez A, Trelles O, Carazo J, Pascual-Montano A: Integrated analysis of gene expression by association rules discovery. BMC Bioinformatics 2006, 7:1-16.

27. Henriques R, Madeira SC: BiP: effective discovery of overlapping biclusters using flexible plaid models. In BIOKDD, ACM SIGKDD. New York, NY, USA: ACM; 2014

28. Henriques R, Madeira S: BicSPAM: flexible biclustering using sequential patterns. BMC Bioinformatics 2014, 15:130.

29. Agrawal $R$, Imieliński T, Swami A: Mining association rules between sets of items in large databases. SIGMOD Rec 1993, 22(2):207-216. 
30. Bellay J, Atluri G, Sing TL, Toufighi K, Costanzo M, Ribeiro PSM, Pandey G, Baller J, VanderSluis B, Michaut M, Han S, Kim P, Brown G, Andrews B, Boone $C$, Kumar $V$, Myers $C$ : Putting genetic interactions in context through a global modular decomposition. Genome Res 2011, 21(8):1375-1387.

31. Uno T, Kiyomi M, Arimura H: LCM ver.3: collaboration of array, bitmap and prefix tree for frequent itemset mining. In OSDM. Chicago, Illinois: ACM; 2005:77-86.

32. Burdick D, Calimlim M, Gehrke J: MAFIA: a maximal frequent itemset algorithm for transactional databases. In ICDE. Heidelberg, Germany: IEEE CS; 2001:443-452.

33. Pasquier $N$, Bastide $Y$, Taouil R, Lakhal L: Efficient mining of association rules using closed itemset lattices. Inf Syst 1999, 24:25-46.

34. Mahfouz M, Ismail M: BIDENS: Iterative density based biclustering algorithm with application to gene expression analysis. In World Acad. of Science, Eng. and Tech., Volume 37. Riverside, USA: WASET.org; 2009:342-348.

35. Alves R, Rodríguez-Baena DS, Aguilar-Ruiz JS: Gene association analysis: a survey of frequent pattern mining from gene expression data. Brief Bioinformatics 2010, 11(2):210-224.

36. Atluri G, Bellay J, Pandey G, Myers C, Kumar V: Discovering coherent value bicliques in genetic interaction data. In BIOKDD: ACM; 2000.

37. Gupta R, Rao N, Kumar V: Discovery of error-tolerant biclusters from noisy gene expression data. BMC Bioinformatics 2011, 12(12):1-17.

38. Huang $Y$, Xiong $H$, Wu W, Sung SY: Mining quantitative maximal hyperclique patterns: a summary of results. In Proceedings of the 10th Pacific-Asia conference on Advances in Knowledge Discovery and Data Mining, PAKDD'06. Berlin: Heidelberg: Springer-Verlag; 2006:552-556.

39. Steinbach $M$, Tan PN, Xiong $H$, Kumar V: Generalizing the notion of support. In Proceedings of the tenth ACM SIGKDD international conference on Knowledge discovery and data mining, KDD '04. New York, NY, USA ACM; 2004:689-694

40. Han EH, Karypis G, Kumar V: Min-apriori: An algorithm for finding association rules in data with continuous attributes. Department of Computer Science. University of Minnesota, Minneapolis 1997.

41. Agrawal R, Srikant R: Fast algorithms for mining association rules in large databases. In VLDB. San Francisco, USA: Morgan Kaufmann; 1994:487-499.

42. Han J, Pei J, Yin Y: Mining frequent patterns without candidate generation. SIGMOD ReC 2000, 29(2):1-12.

43. Zaki MJ, Gouda K: Fast vertical mining using diffsets. In KDD. New York, NY, USA: ACM; 2003:326-335.

44. Henriques R, Madeira SC, Antunes C: F2G: efficient discovery of full-patterns. In ECML/PKDD nfMCP. Prague: Springer Verlag; 2013.

45. Zaki MJ, Hsiao CJ: Efficient algorithms for mining closed itemsets and their lattice structure. IEEE TKDE 2005, 17(4):462-478.

46. Pan F, Cong G, Tung AKH, Yang J, Zaki MJ: Carpenter: finding closed patterns in long biological datasets. In KDD. Washington, DC, USA: ACM; 2003:637-642

47. Pan F, Tung A, Cong G, Xu X: COBBLER: combining column and row enumeration for closed pattern discovery. In SSDM. Santorini Island, Greece: IEEE; 2004:21-30.

48. de Souto M, de Araujo D, Costa I, Soares R, Ludermir T, Schliep A: Comparative study on normalization procedures for cluster analysis of gene expression datasets. In IJCNN. Hong Kong, China: IEEE; 2008:2792-2798.

49. Xin D, Cheng $H$, Yan $X$, Han J: Extracting redundancy-aware top-k patterns. In Proceedings of the 12th ACM SIGKDD International Conference on Knowledge Discovery and Data Mining, KDD '06. New York, NY, USA: ACM; 2006:444-453.

50. Yan $\mathrm{X}$, Cheng $H$, Han J, Xin D: Summarizing itemset patterns: a profile-based approach. In Proceedings of the Eleventh ACM SIGKDD International Conference on Knowledge Discovery in Data Mining, KDD '05. New York, NY, USA: ACM; 2005:314-323.

51. Troyanskaya O, Cantor M, Sherlock G, Brown P, Hastie T, Tibshirani R, Botstein D, Altman RB: Missing value estimation methods for DNA microarrays. Bioinformatics 2001, 17(6):520-525.

52. Donders A, van der Heijden G, Stijnen T, Moons K: Review: a gentle introduction to imputation of missing values. Clinical epidemiology 2006, 59(10):1087-91.

53. Hellem T, Dysvik B, Jonassen I: LSimpute: accurate estimation of missing values in microarray data with least squares methods. Nucleic Acids Res 2004, 32(3):34+
54. http://cran.r-project.org/doc/contrib/Ricci-distributions-en.pdf (accessed 11 Nov 2014)

55. Ramesh G, Maniatty WA, Zaki MJ: Feasible itemset distributions in data mining: theory and application. In Symposium on Princ. of data. sys., San Diego, USA: ACM Press; 2003:284-295.

56. Assent I, Krieger R, Muller E, Seidl T: DUSC: dimensionality unbiased subspace clustering. In ICDM; 2007

57. Sequeira K, Zaki M: SCHISM: a new approach to interesting subspace mining. Int J Bus Intell Data Min 2005, 1(2):137-160.

58. Prelić A, Bleuler S, Zimmermann P, Wille A, Bühlmann P, Gruissem W Hennig $L$, Thiele $L$, Zitzler E: A systematic comparison and evaluation of biclustering methods for gene expression data. Bioinf 2006 22(9):1122-1129.

59. Bozdağ D, Kumar AS, Catalyurek UV: Comparative analysis of biclustering algorithms. In BCB. New York, NY, USA: ACM; 2010:265-274.

60. Patrikainen A, Meila M: Comparing subspace clusterings. IEEE TKDE 2006, 18(7):902-916

61. Munkres J: Algorithms for the assignment and transportation problems. Soc Ind App/ Math 1957, 5:32-38.

62. Cheng Y, Church GM: Biclustering of expression data. In Intelligent Systems for Molecular Biology: AAAI Press; 2000:93-103.

63. Berriz GF, King OD, Bryant B, Sander C, Roth FP: Characterizing gene sets with FuncAssociate. Bioinformatics 2003, 19:2502-2504

64. http://www.bioinf.jku.at/software/fabia/benchmark.html.

65. http://web.ist.utl.pt/rmch/software/bicpam/

66. Pontes B, Giráldez R, Aguilar-Ruiz JS: Configurable pattern-based evolutionary biclustering of gene expression data. Algorithms Mol Biol 2013, 8:4

67. Ihmels J, Bergmann S, Barkai N: Defining transcription modules using large-scale gene expression data. Bioinformatics 2004, 20(13):1993-2003

68. http://www.bioinf.jku.at/software/fabia/fabia.html.

69. http://cran.r-project.org/web/packages/biclust.

70. Barkow S, Bleuler S, Prelić A, Zimmermann P, Zitzler E: BicAT: a biclustering analysis toolbox. Bioinformatics 2006, 22(10):1282-1283.

71. http://acgt.cs.tau.ac.il/expander.

72. http://www.philippe-fournier-viger.com/spmf/

73. Madeira S, Teixeira MNPC, Sá-Correia I, Oliveira A: Identification of regulatory modules in time series gene expression data using a linear time biclustering algorithm. IEEE/ACM Trans Comput Biol Bioinformatics 2010, 1:153-165.

74. http://www.bioinf.jku.at/software/fabia/gene_expression.html.

75. http://chemogenomics.stanford.edu/supplements/03nuc/datasets.html.

76. Rosenwald A, dlb/c team: The use of molecular profiling to predict survival after chemotherapy for diffuse large-B-cell lymphoma. $N$ Engl J Med 2002, 346(25):1937-1947.

77. Lee W, Tillo D, Bray N, Morse RH, Davis RW, Hughes TR, Nislow C: A high-resolution atlas of nucleosome occupancy in yeast. Nat Genet 2007, 39(10):1235-1244

78. Gasch AP, Spellman PT, Kao CM, Carmel-Harel O, Eisen MB, Storz G, Botstein D, Brown PO: Genomic expression programs in the response of yeast cells to environmental changes. Mol Biol Cell 2000, 11(12):4241-4257

79. Martin D, Brun C, Remy E, Mouren P, Thieffry D, Jacq B: GOToolBox: functional analysis of gene datasets based on Gene Ontology. Genome Biol 2004, 12:101

80. http://web.ist.utl.pt/rmch/software/bicpam/

81. Wlodkowic D, Skommer J, McGuinness D, Hillier C, Darzynkiewicz Z: ER-Golgi network-A future target for anti-cancer therapy. Leuk Res 2009, 33(11):1440-1447

82. Bracken AP, Bond U: Reassembly and protection of small nuclear ribonucleoprotein particles by heat shock proteins in yeast cells. Rna 1999, 5(12):1586-1596.

83. Lee AP, Yang Y, Brenner S, Venkatesh B: TFCONES: a database of vertebrate transcription factor-encoding genes and their associated conserved noncoding elements. BMC Genomics 2007, 8:441.

84. Teixeira M, Monteiro P, Guerreiro J, Gonçalves J, Mira N, dos Santos S, Cabrito T, Palma M, Costa C, Francisco A, Madeira S, Oliveira A, Freitas A, Sá-Correia I: The YEASTRACT database: an upgraded information system for the analysis of gene and genomic transcription regulation in Saccharomyces cerevisiae. Nucleic Acids Res 2014. (Database issue). 
85. Safran M, Dalah I, Alexander J, Rosen N, Stein TI, Shmoish M, Nativ N, Bahir I, Doniger T, Krug H, et al: GeneCards Version 3: the human gene integrator. Database 2010, 2010:baq020.

86. Cherry JM, Hong EL, Amundsen C, Balakrishnan R, Binkley G, Chan ET, Christie KR, Costanzo MC, Dwight SS, Engel SR, et al: Saccharomyces genome Database: the genomics resource of budding yeast. Nucleic Acids Res 2011:gkr1029.

87. Nakagawa Y, Sakumoto N, Kaneko Y, Harashima S: Mga2p is a putative sensor for low temperature and oxygen to induce ole1 transcription in saccharomyces cerevisiae. Biochem Biophys Res Commun 2002, 291(3):707-713.

88. Doolin MT, Johnson AL, Johnston LH, Butler G: Overlapping and distinct roles of the duplicated yeast transcription factors Ace2p and Swi5p. Mol Microbiol 2001, 40(2):422-432.

Submit your next manuscript to BioMed Central and take full advantage of:

- Convenient online submission

- Thorough peer review

- No space constraints or color figure charges

- Immediate publication on acceptance

- Inclusion in PubMed, CAS, Scopus and Google Scholar

- Research which is freely available for redistribution

Submit your manuscript at www.biomedcentral.com/submit
( Biomed Central 